\title{
Measurement of $\bar{\nu}_{\mu}$ and $\nu_{\mu}$ charged current inclusive cross sections and their ratio with the $\mathrm{T} 2 \mathrm{~K}$ off-axis near detector
}

K. Abe ${ }^{45}$ J. Amey, ${ }^{15}$ C. Andreopoulos,${ }^{43,25}$ M. Antonova, ${ }^{20}$ S. Aoki, ${ }^{22}$ A. Ariga, ${ }^{1}$ Y. Ashida, ${ }^{23}$ S. Ban, ${ }^{23}$ M. Barbi ${ }^{37}$ G. J. Barker, ${ }^{53}$ G. Barr, ${ }^{33}$ C. Barry, ${ }^{25}$ M. Batkiewicz, ${ }^{11}$ V. Berardi, ${ }^{16}$ S. Berkman ${ }^{3,49}$ S. Bhadra, ${ }^{57}$ S. Bienstock, ${ }^{34}$ A. Blondel,${ }^{10}$ S. Bolognesi, ${ }^{5}$ S. Bordoni, ${ }^{13,}{ }^{*}$ S. B. Boyd,${ }^{53}$ D. Brailsford ${ }^{24}$ A. Bravar, ${ }^{10}$ C. Bronner,${ }^{45}$

M. Buizza Avanzini, ${ }^{9}$ R. G. Calland, ${ }^{21}$ T. Campbell, ${ }^{7}$ S. Cao, ${ }^{12}$ S. L. Cartwright, ${ }^{41}$ M. G. Catanesi,${ }^{16}$ A. Cervera ${ }^{14}$ A. Chappell, ${ }^{53}$ C. Checchia, ${ }^{18}$ D. Cherdack,${ }^{7}$ N. Chikuma,${ }^{44}$ G. Christodoulou, ${ }^{25}$ J. Coleman, ${ }^{25}$ G. Collazuol, ${ }^{18}$

D. Coplowe, ${ }^{33}$ A. Cudd,${ }^{27}$ A. Dabrowska, ${ }^{11}$ G. De Rosa,${ }^{17}$ T. Dealtry, ${ }^{24}$ P. F. Denner, ${ }^{53}$ S. R. Dennis, ${ }^{25}$ C. Densham, ${ }^{43}$ F. Di Lodovico, ${ }^{36}$ S. Dolan, ${ }^{33}$ O. Drapier, ${ }^{9}$ K. E. Duffy, ${ }^{33}$ J. Dumarchez, ${ }^{34}$ P. Dunne, ${ }^{15}$ S. Emery-Schrenk, ${ }^{5}$

A. Ereditato, ${ }^{1}$ T. Feusels, ${ }^{3,49}$ A. J. Finch, ${ }^{24}$ G. A. Fiorentini, ${ }^{57}$ M. Friend, ${ }^{12, \dagger}$ Y. Fujiii, ${ }^{12, \dagger}$ D. Fukuda, ${ }^{31}$ Y. Fukuda, ${ }^{28}$ A. Garcia, ${ }^{13}$ C. Giganti, ${ }^{34}$ F. Gizzarelli, ${ }^{5}$ T. Golan, ${ }^{55}$ M. Gonin, ${ }^{9}$ D. R. Hadley, ${ }^{53}$ L. Haegel, ${ }^{10}$ J. T. Haigh ${ }^{53}$ D. Hansen, ${ }^{35}$ J. Harada, ${ }^{32}$ M. Hartz, ${ }^{21,49}$ T. Hasegawa, ${ }^{12, \dagger}$ N. C. Hastings, ${ }^{37}$ T. Hayashino, ${ }^{23}$ Y. Hayato, ${ }^{45,21}$ A. Hillairet, ${ }^{50}$ T. Hiraki, ${ }_{45}^{23}$ A. Hiramoto, ${ }^{23}$ S. Hirota, ${ }^{23}$ M. Hogan, ${ }^{7}$ J. Holeczek, ${ }^{42}$ F. Hosomi, ${ }^{44}$ K. Huang, ${ }^{23}$ A. K. Ichikawa, ${ }^{23}$ M. Ikeda, ${ }^{45}$ J. Imber, ${ }^{9}$ J. Insler, ${ }^{26}$ R. A. Intonti, ${ }^{16}$ T. Ishida, ${ }^{12, \dagger}$ T. Ishii, ${ }^{12, \dagger}$ E. Iwai, ${ }^{12}$ K. Iwamoto, ${ }^{44}$ A. Izmaylov, ${ }^{14,20}$ B. Jamieson, ${ }^{54}$ M. Jiang, ${ }^{23}$ S. Johnson, ${ }^{6}$ P. Jonsson, ${ }^{15}$ C. K. Jung, ${ }^{30,}$ M. Kabirnezhad, ${ }^{29}$ A. C. Kaboth, ${ }^{39,43}$ T. Kajita, ${ }^{46, *}$ H. Kakuno, ${ }^{47}$ J. Kameda, ${ }^{45}$ D. Karlen, ${ }^{50,49}$ T. Katori, ${ }^{36}$ E. Kearns, $, 21, \sharp$ M. Khabibullin, ${ }^{20}$ A. Khotjantsev, ${ }^{20}$ H. Kim, ${ }^{32}$ J. Kim, ${ }^{3,49}$ S. King, ${ }^{36}$ J. Kisiel, ${ }^{42}$ A. Knight, ${ }^{53}$ A. Knox,${ }^{24}$ T. Kobayashi, ${ }^{12, \dagger}$ L. Koch, ${ }^{40}$ T. Koga, ${ }^{44}$ P. P. Koller, ${ }^{1}$ A. Konaka, ${ }^{49}$ L. L. Kormos, ${ }^{24}$ Y. Koshio, ${ }^{31,}$ K. Kowalik, ${ }^{29}$ Y. Kudenko, ${ }^{20,8}$ R. Kurjata, ${ }^{52}$ T. Kutter, ${ }^{26}$ J. Lagoda, ${ }^{29}$ I. Lamont, ${ }^{24}$ M. Lamoureux, ${ }^{5}$ P. Lasorak, ${ }^{36}$ M. Laveder, ${ }^{18}$ M. Lawe, ${ }^{24}$ M. Licciardi, ${ }^{9}$ T. Lindner, ${ }^{49}$ Z. J. Liptak, ${ }^{6}$ R. P. Litchfield, ${ }^{15}$ X. Li ${ }^{30}$ A. Longhin, ${ }^{18}$ J. P. Lopez, ${ }^{6}$ T. Lou, ${ }^{44}$ L. Ludovici, ${ }^{19}$ X. Lu, ${ }^{33}$ L. Magaletti, ${ }^{16}$ K. Mahn, ${ }^{27}$ M. Malek, ${ }^{41}$ S. Manly, ${ }^{38}$ L. Maret, ${ }^{10}$ A. D. Marino, ${ }^{6}$ J. F. Martin ${ }^{48}$ P. Martins, ${ }^{36}$ S. Martynenko, ${ }^{30}$ T. Maruyama, ${ }^{12, \dagger}$ V. Matveev, ${ }^{20}$ K. Mavrokoridis ${ }^{25}$ W. Y. Ma, ${ }^{15}$ E. Mazzucato, ${ }^{5}$ M. McCarthy, ${ }^{57}$ N. McCauley, ${ }^{25}$ K. S. McFarland, ${ }^{38}$ C. McGrew, ${ }^{30}$ A. Mefodiev, ${ }^{20}$ C. Metelko, ${ }^{25}$ M. Mezzetto, ${ }^{18}$ A. Minamino, ${ }^{56}$ O. Mineev, ${ }^{20}$ S. Mine, ${ }^{4}$ A. Missert, ${ }^{6}$ M. Miura, ${ }^{45}$ S. Moriyama, ${ }^{45,}$ J. Morrison, ${ }^{27}$ Th. A. Mueller, ${ }^{9}$ T. Nakadaira, ${ }^{1, \dagger}$ M. Nakahata, ${ }^{45,21}$ K. G. Nakamura, ${ }^{23}$ K. Nakamura, ${ }^{21,12, \dagger}$ K. D. Nakamura, ${ }^{23}$ Y. Nakanishi, ${ }^{23}$ S. Nakayama, ${ }^{45, \$}$ T. Nakaya, ${ }^{23,21}$ K. Nakayoshi, ${ }^{12, \dagger}$ C. Nantais, ${ }^{48}$ C. Nielsen, ${ }^{3,49}$ K. Nishikawa, ${ }^{2, \dagger}$ Y. Nishimura, ${ }^{46}$ P. Novella, ${ }^{14}$ J. Nowak, ${ }^{24}$ H. M. O'Keeffe, ${ }^{24}$ K. Okumura, ${ }^{46,21}$ T. Okusawa, ${ }^{32}$ W. Oryszczak, ${ }^{51}$ S. M. Oser,${ }^{3,49}$ T. Ovsyannikova, ${ }^{20}$ R. A. Owen, ${ }^{36}$ Y. Oyama, ${ }^{12, \dagger}$ V. Palladino, ${ }^{17}$ J. L. Palomino, ${ }^{30}$ V. Paolone, ${ }^{35}$ N. D. Patel,,${ }^{23}$ P. Paudyal, ${ }^{25}$ M. Pavin ${ }^{34}$ D. Payne, ${ }^{25}$ Y. Petrov,${ }^{3,49}$ L. Pickering, ${ }^{15}$ E. S. Pinzon Guerra, ${ }^{57}$ C. Pistillo, ${ }^{1}$ B. Popov, ${ }^{34, \|}$ M. Posiadala-Zezula, ${ }^{51}$ J.-M. Poutissou, ${ }^{49}$ A. Pritchard, ${ }^{25}$ P. Przewlocki, ${ }^{29}$ B. Quilain, ${ }^{23}$ T. Radermacher, ${ }^{40}$ E. Radicioni, ${ }^{16}$ P. N. Ratoff, ${ }^{24}$ M. A. Rayner, ${ }^{10}$ E. Reinherz-Aronis, ${ }^{7}$ C. Riccio, ${ }^{17}$ E. Rondio, ${ }^{29}$ B. Rossi ${ }^{17}$ S. Roth ${ }^{40}$ A. C. Ruggeri, ${ }^{17}$ A. Rychter ${ }^{52}$ K. Sakashita, ${ }^{12,}{ }^{\dagger}$ F. Sánchez, ${ }^{13}$ E. Scantamburlo, ${ }^{10}$ K. Scholberg, ${ }^{8,}$ J. Schwehr, ${ }^{7}$ M. Scott, ${ }^{49}$ Y. Seiya, ${ }^{32}$ T. Sekiguchi, ${ }^{12, \dagger}$ H. Sekiya, ${ }^{45,21, \sharp}$ D. Sgalaberna, ${ }^{10}$ R. Shah, ${ }^{43,33}$ A. Shaikhiev, ${ }^{20}$ F. Shaker, ${ }^{54}$ D. Shaw, ${ }^{24}$ M. Shiozawa, ${ }^{45,21}$ T. Shirahige, ${ }^{31}$ M. Smy, ${ }^{4}$ J. T. Sobczyk ${ }^{55}$ H. Sobel, ${ }^{4,21}$ J. Steinmann, ${ }^{40}$ T. Stewart, ${ }^{43}$ P. Stowell, ${ }^{41}$ Y. Suda, ${ }^{44}$ S. Suvorov ${ }^{20}$ A. Suzuki, ${ }^{22}$ S. Y. Suzuki, ${ }^{12,},{ }^{\dagger}$ Y. Suzuki, ${ }^{21}$ R. Tacik, ${ }^{37,49}$ M. Tada, ${ }^{12, \uparrow}$ A. Takeda, ${ }^{45}$ Y. Takeuchi, ${ }^{22,21}$ R. Tamura, ${ }^{44}$ H. K. Tanaka, ${ }^{45, \sharp}$ H. A. Tanaka, ${ }^{48,49,}$, T. Thakore, ${ }^{26}$ L.F. Thompson, ${ }^{41}$ S. Tobayama, ${ }^{3,49}$ W. Toki, ${ }^{7}$ T. Tomura, ${ }^{45}$ T. Tsukamoto, ${ }^{12, \dagger}$ M. Tzanov, ${ }^{26}$ M. Vagins, ${ }^{21,4}$ Z. Vallari, ${ }^{30}$ G. Vasseur, ${ }^{5}$ C. Vilela, ${ }^{30}$ T. Vladisavljevic, ${ }^{33,21}$ T. Wachala, ${ }^{11}$ C. W. Walter, ${ }^{8, *}$ D. Wark, ${ }^{43,33}$ M. O. Wascko, ${ }^{15}$ A. Weber, ${ }^{43,33}$ R. Wendell, ${ }^{23, \$}$ M. J. Wilking, ${ }^{30}$ C. Wilkinson, ${ }^{1}$ J. R. Wilson, ${ }^{36}$ R. J. Wilson, ${ }^{7}$ C. Wret, ${ }^{15}$ Y. Yamada, ${ }^{12 \dagger}$ K. Yamamoto, ${ }^{32}$ C. Yanagisawa, ${ }^{30,}{ }^{, *}{ }^{\prime}$ T. Yano, ${ }^{22}$ S. Yen, ${ }^{49}$ N. Yershov, ${ }^{20}$ M. Yokoyama, ${ }^{44, \$}$ M. Yu, ${ }^{57}$ A. Zalewska, ${ }^{11}$ J. Zalipska, ${ }^{29}$ L. Zambelli, ${ }^{12, \dagger}$ K. Zaremba, ${ }^{52}$ M. Ziembicki, ${ }^{52}$ E. D. Zimmerman, ${ }^{6}$ and M. Zito ${ }^{5}$

(The T2K Collaboration)

\footnotetext{
${ }^{1}$ University of Bern, Albert Einstein Center for Fundamental Physics, Laboratory for High Energy Physics (LHEP), Bern, Switzerland

${ }^{2}$ Boston University, Department of Physics, Boston, Massachusetts, USA

${ }^{3}$ University of British Columbia, Department of Physics and Astronomy, Vancouver, British Columbia, Canada

${ }^{4}$ University of California, Irvine, Department of Physics and Astronomy, Irvine, California, USA ${ }^{5}$ IRFU, CEA Saclay, Gif-sur-Yvette, France

${ }^{6}$ University of Colorado at Boulder, Department of Physics, Boulder, Colorado, USA

${ }^{7}$ Colorado State University, Department of Physics, Fort Collins, Colorado, USA
} 
${ }^{8}$ Duke University, Department of Physics, Durham, North Carolina, USA

${ }^{9}$ Ecole Polytechnique, IN2P3-CNRS, Laboratoire Leprince-Ringuet, Palaiseau, France

${ }^{10}$ University of Geneva, Section de Physique, DPNC, Geneva, Switzerland

${ }^{11}$ H. Niewodniczanski Institute of Nuclear Physics PAN, Cracow, Poland

${ }^{12}$ High Energy Accelerator Research Organization (KEK), Tsukuba, Ibaraki, Japan

${ }^{13}$ Institut de Fisica d'Altes Energies (IFAE), The Barcelona Institute of Science and Technology, Campus $U A B$, Bellaterra (Barcelona) Spain

${ }^{14}$ IFIC (CSIC \& University of Valencia), Valencia, Spain

${ }^{15}$ Imperial College London, Department of Physics, London, United Kingdom

${ }^{16}$ INFN Sezione di Bari and Università e Politecnico di Bari,

Dipartimento Interuniversitario di Fisica, Bari, Italy

${ }^{17}$ INFN Sezione di Napoli and Università di Napoli, Dipartimento di Fisica, Napoli, Italy

${ }^{18}$ INFN Sezione di Padova and Università di Padova, Dipartimento di Fisica, Padova, Italy

${ }^{19}$ INFN Sezione di Roma and Università di Roma "La Sapienza," Roma, Italy

${ }^{20}$ Institute for Nuclear Research of the Russian Academy of Sciences, Moscow, Russia

${ }^{21}$ Kavli Institute for the Physics and Mathematics of the Universe (WPI),

The University of Tokyo Institutes for Advanced Study, University of Tokyo, Kashiwa, Chiba, Japan

${ }^{22}$ Kobe University, Kobe, Japan

${ }^{23}$ Kyoto University, Department of Physics, Kyoto, Japan

${ }^{24}$ Lancaster University, Physics Department, Lancaster, United Kingdom

${ }^{25}$ University of Liverpool, Department of Physics, Liverpool, United Kingdom

${ }^{26}$ Louisiana State University, Department of Physics and Astronomy, Baton Rouge, Louisiana, USA

${ }^{27}$ Michigan State University, Department of Physics and Astronomy, East Lansing, Michigan, USA

${ }^{28}$ Miyagi University of Education, Department of Physics, Sendai, Japan

${ }^{29}$ National Centre for Nuclear Research, Warsaw, Poland

${ }^{30}$ State University of New York at Stony Brook, Department of Physics and Astronomy, Stony Brook, New York, USA

${ }^{31}$ Okayama University, Department of Physics, Okayama, Japan

${ }^{32}$ Osaka City University, Department of Physics, Osaka, Japan

${ }^{33}$ Oxford University, Department of Physics, Oxford, United Kingdom

${ }^{34}$ UPMC, Université Paris Diderot, CNRS/IN2P3, Laboratoire de Physique Nucléaire et de Hautes Energies (LPNHE), Paris, France

${ }^{35}$ University of Pittsburgh, Department of Physics and Astronomy, Pittsburgh, Pennsylvania, USA

${ }^{36}$ Queen Mary University of London, School of Physics and Astronomy, London, United Kingdom

${ }^{37}$ University of Regina, Department of Physics, Regina, Saskatchewan, Canada

${ }^{38}$ University of Rochester, Department of Physics and Astronomy, Rochester, New York, USA

${ }^{39}$ Royal Holloway University of London, Department of Physics, Egham, Surrey, United Kingdom

${ }^{40}$ RWTH Aachen University, III. Physikalisches Institut, Aachen, Germany

${ }^{41}$ University of Sheffield, Department of Physics and Astronomy, Sheffield, United Kingdom

${ }^{42}$ University of Silesia, Institute of Physics, Katowice, Poland

${ }^{43}$ STFC, Rutherford Appleton Laboratory, Harwell Oxford, and Daresbury Laboratory,

Warrington, United Kingdom

${ }^{44}$ University of Tokyo, Department of Physics, Tokyo, Japan

${ }^{45}$ University of Tokyo, Institute for Cosmic Ray Research,

Kamioka Observatory, Kamioka, Japan

${ }^{46}$ University of Tokyo, Institute for Cosmic Ray Research, Research Center for Cosmic Neutrinos, Kashiwa, Japan

${ }^{47}$ Tokyo Metropolitan University, Department of Physics, Tokyo, Japan

${ }^{48}$ University of Toronto, Department of Physics, Toronto, Ontario, Canada

${ }^{49}$ TRIUMF, Vancouver, British Columbia, Canada

${ }^{50}$ University of Victoria, Department of Physics and Astronomy, Victoria, British Columbia, Canada

${ }^{51}$ University of Warsaw, Faculty of Physics, Warsaw, Poland

${ }^{52}$ Warsaw University of Technology, Institute of Radioelectronics, Warsaw, Poland

${ }^{53}$ University of Warwick, Department of Physics, Coventry, United Kingdom

${ }^{54}$ University of Winnipeg, Department of Physics, Winnipeg, Manitoba, Canada

${ }^{55}$ Wroclaw University, Faculty of Physics and Astronomy, Wroclaw, Poland 


\footnotetext{
${ }^{56}$ Yokohama National University, Faculty of Engineering, Yokohama, Japan

${ }^{57}$ York University, Department of Physics and Astronomy, Toronto, Ontario, Canada
}

(Received 15 June 2017; published 5 September 2017)

\begin{abstract}
We report a measurement of cross section $\sigma\left(\nu_{\mu}+\right.$ nucleus $\left.\rightarrow \mu^{-}+X\right)$ and the first measurements of the cross section $\sigma\left(\bar{\nu}_{\mu}+\right.$ nucleus $\left.\rightarrow \mu^{+}+X\right)$ and their ratio $R\left(\frac{\sigma(\bar{\nu})}{\sigma(\nu)}\right)$ at (anti) neutrino energies below $1.5 \mathrm{GeV}$. We determine the single momentum bin cross section measurements, averaged over the $\mathrm{T} 2 \mathrm{~K} \bar{\nu} / \nu$-flux, for the detector target material (mainly carbon, oxygen, hydrogen and copper) with phase space restricted laboratory frame kinematics of $\theta_{\mu}<32^{\circ}$ and $p_{\mu}>500 \mathrm{MeV} / c$. The results are $\sigma(\bar{\nu})=$ $(0.900 \pm 0.029$ (stat) \pm 0.088 (syst) $) \times 10^{-39}$ and $\sigma(\nu)=(2.41 \pm 0.022$ (stat) $\pm 0.231($ syst $)) \times 10^{-39}$ in units of $\mathrm{cm}^{2} /$ nucleon and $R\left(\frac{\sigma(\bar{\nu})}{\sigma(\nu)}\right)=0.373 \pm 0.012$ (stat) \pm 0.015 (syst).
\end{abstract}

DOI: 10.1103/PhysRevD.96.052001

\section{INTRODUCTION}

Since the 1998 discovery [1] of neutrino oscillations, there have been major advances in neutrino disappearance and appearance oscillation measurements, and all the fundamental neutrino mixing parameters [2] have been determined except for the mass hierarchy and the chargeparity $(C P)$ phase $\delta_{C P}$. Evidence of $\delta_{C P} \neq 0, \pi$ leads to the nonconservation or violation of the charge-parity symmetry (CPV). This is tested by measuring the neutrino $\nu_{\mu} \rightarrow \nu_{e}$ and antineutrino $\bar{\nu}_{\mu} \rightarrow \bar{\nu}_{e}$ appearance oscillation event rates to determine if the neutrino and antineutrino oscillation appearance probabilities, $P\left(\nu_{\mu} \rightarrow \nu_{e}\right)$ and $\bar{P}\left(\bar{\nu}_{\mu} \rightarrow \bar{\nu}_{e}\right)$ are equal in vacuum [2,3] at the same ratio of the oscillation distance $L$ over the neutrino energy $E$ or $\frac{L}{E}$. Major long-baseline neutrino experiments [4] have been built and future projects [5] are proposed to determine these probabilities using separate $\nu_{\mu}$ and $\bar{\nu}_{\mu}$ beams that cross near and far detectors. The probabilities are obtained from near detector measurements of the $\nu_{\mu}+N$ and $\bar{\nu}_{\mu}+N$ charged current (CC) interactions and cross sections, where $N$ is the target nucleon, and far detector measurements of $\nu_{e}+N$ and $\bar{\nu}_{e}+N$ CC interactions.

\footnotetext{
*Now at CERN.

Also at J-PARC, Tokai, Japan.

${ }^{*}$ Affiliated member at Kavli IPMU (WPI), the University of Tokyo, Japan.

${ }^{\S}$ Also at National Research Nuclear University "MEPhI" and Moscow Institute of Physics and Technology, Moscow, Russia.

"Also at JINR, Dubna, Russia.

"Also at Institute of Particle Physics, Canada.

** Also at BMCC/CUNY, Science Department, New York, New York, USA.

Published by the American Physical Society under the terms of the Creative Commons Attribution 4.0 International license. Further distribution of this work must maintain attribution to the author(s) and the published article's title, journal citation, and DOI.
}

In this paper, the $\mathrm{T} 2 \mathrm{~K}$ Collaboration, using the off-axis near detector (ND280), presents a measurement at a peak energy $\sim 0.6 \mathrm{GeV}$ of the charged current inclusive (CCINC) $\nu_{\mu}+N$ cross section and first CCINC measurements of the $\bar{\nu}_{\mu}+N$ cross section and their ratio of the $\bar{\nu}_{\mu}+N$ over the $\nu_{\mu}+N$ CCINC cross section. These $\nu_{\mu}$ and $\bar{\nu}_{\mu}$ measurements are important to understand their impact on future CPV measurements and to test neutrino cross section models.

$\mathrm{T} 2 \mathrm{~K}$ has published flux averaged neutrino-mode measurements of CCINC [6] and charged current quasi-elastic like (CCQE) [7] cross sections per nucleon of (6.91 \pm 0.13 (stat) \pm 0.84 (syst) $) \times 10^{-39} \mathrm{~cm}^{2}$ and $(4.15 \pm 0.6) \times$ $10^{-39} \mathrm{~cm}^{2}$, respectively. These measurements were performed using the Fine-Grain Detector (FGD) which has different detector systematics compared to the measurements presented in this paper. There are no published CCINC $\bar{\nu}_{\mu}$ measurements at energies below $1.5 \mathrm{GeV}$; however, the MINVERVA Collaboration recently published [8] CCINC results above $2 \mathrm{GeV}$ and the MiniBooNE Collaboration has published [9] CCQE measurements in both $\bar{\nu}_{\mu}$ and $\nu_{\mu}$ modes which require larger axial mass values compared to other experiments to fit their observed data. There are several multinucleon models (2 particle 2 hole, or $2 \mathrm{p} 2 \mathrm{~h}$ ) [10-12] proposed to explain large cross sections. In addition, in some models it has been predicted [10] that the difference between the $\nu_{\mu}$ and $\bar{\nu}_{\mu}$ cross sections is expected to increase when $2 \mathrm{p} 2 \mathrm{~h}$ effects [13] are included. The measurements of the ratio, sum, and difference of these cross sections, which have very different systematic errors, will be presented.

Following this introduction, the paper is organized as follows. We begin with a description of the ND280 off-axis detector and the neutrino beam in Sec. II. Then the Monte Carlo (MC) simulation is presented in Sec. III, followed by the event selection given in Sec. IV. The analysis methods and systematic error evaluations are presented in Secs. V and VI, and we finally conclude with the results and conclusions in Secs. VII and VIII. 


\section{BEAM AND DETECTOR}

The T2K experiment [14] is composed of a neutrino beam line and a near detector at the J-PARC laboratory in Tokai, Japan, and the far detector Super-Kamiokande (SK) situated $295 \mathrm{~km}$ away in the Kamioka mine. The J-PARC accelerator complex produces a $30 \mathrm{GeV}$ energy proton beam with spills every $2.48 \mathrm{~s}$ that contain eight beam bunches which are $580 \mathrm{~ns}$ apart. At this spill and repetition rate, a beam power of $430 \mathrm{~kW}$ produces $2.25 \times 10^{14}$ protons on target (PoT) per spill corresponding to $\approx 0.8 \times 10^{19}$ PoT integrated per day of data taking.

The proton beam strikes a graphite target to produce pions and kaons that are focused by three magnetic horns into a $96 \mathrm{~m}$ long decay pipe. The polarity of the magnetic horns can be changed to forward horn current (FHC) or reverse horn current (RHC) to select either positive or negative pions and kaons to produce a predominantly $\nu_{\mu}$ or an $\bar{\nu}_{\mu}$ beam. The resulting main neutrino beam axis is parallel to the proton beam direction. SK lies $2.5^{\circ}$ off-axis with respect to the main neutrino beam direction and this arrangement produces at SK both the $\nu_{\mu}$ and $\bar{\nu}_{\mu}$ energies that peak at $\sim 0.6 \mathrm{GeV}$. This $\nu_{\mu}\left(\bar{\nu}_{\mu}\right)$ peak energy with a $295 \mathrm{~km}$ baseline distance, produces an $\frac{L}{E}$ value that maximizes the $\nu_{e}\left(\bar{\nu}_{e}\right)$ appearance rate and has a $\nu_{\mu}\left(\bar{\nu}_{\mu}\right)$ disappearance that minimizes the $\nu_{\mu}\left(\bar{\nu}_{\mu}\right)$ rates at SK.

The ND280 $\nu_{\mu}$ and $\bar{\nu}_{\mu}$ fluxes were determined by simulation of the T2K neutrino beam line [15] using FLUKA2011 [16], GEANT [17], and GCALOR [18] software packages. The simulated hadronic yields have been re-weighted using the NA61/SHINE [19] thin-target data, which has reduced the flux uncertainties to less than $10 \%$ around the flux peak. Detailed descriptions of the ND280 flux uncertainties have been published in previous ND280 analyses [20]. The typical fractional covariance error of the $\mathrm{T} 2 \mathrm{~K} \nu_{\mu}$ and $\bar{\nu}_{\mu}$ fluxes are $\sim 10 \%$ and the $\nu_{\mu}-\bar{\nu}_{\mu}$ correlated flux errors are $\sim 6 \%$. The $\nu_{\mu}$ and $\bar{\nu}_{\mu}$ flux rates per $\mathrm{cm}^{2} / 50 \mathrm{MeV} / 10^{21}$ PoT are plotted in Fig. 1 with superimposed neutral lepton flavors, $\nu_{\mu}, \nu_{e}, \bar{\nu}_{\mu}$ and $\bar{\nu}_{e}$.

The near detector complex, located $280 \mathrm{~m}$ downstream of the target, consists of an on-axis detector (INGRID) and the ND280 off-axis detector. ND280 is positioned inline between the neutrino beam target and SK. The ND280 detector consists of subdetectors inside the refurbished UA1/NOMAD magnet that operates at a $0.2 \mathrm{~T}$ magnetic field whose direction is horizontal and perpendicular to the neutrino beam. The ND280 subdetectors include $\pi^{0}$ detector [21] (PØD), three tracking time projection chambers [22] (TPC1,2,3), two fine-grained detectors (FGD1,2) interleaved with TPC1,2,3, and an electromagnetic calorimeter (ECAL), that encloses the $\mathrm{P} \emptyset \mathrm{D}, \mathrm{TPC} 1-3$ and FGD1-2 subdetectors.

The measurements in this paper used the PøD and the TPC tracking subdetectors in the ND280 detector complex. In our description, the $+\mathrm{Z}$ direction is parallel to the
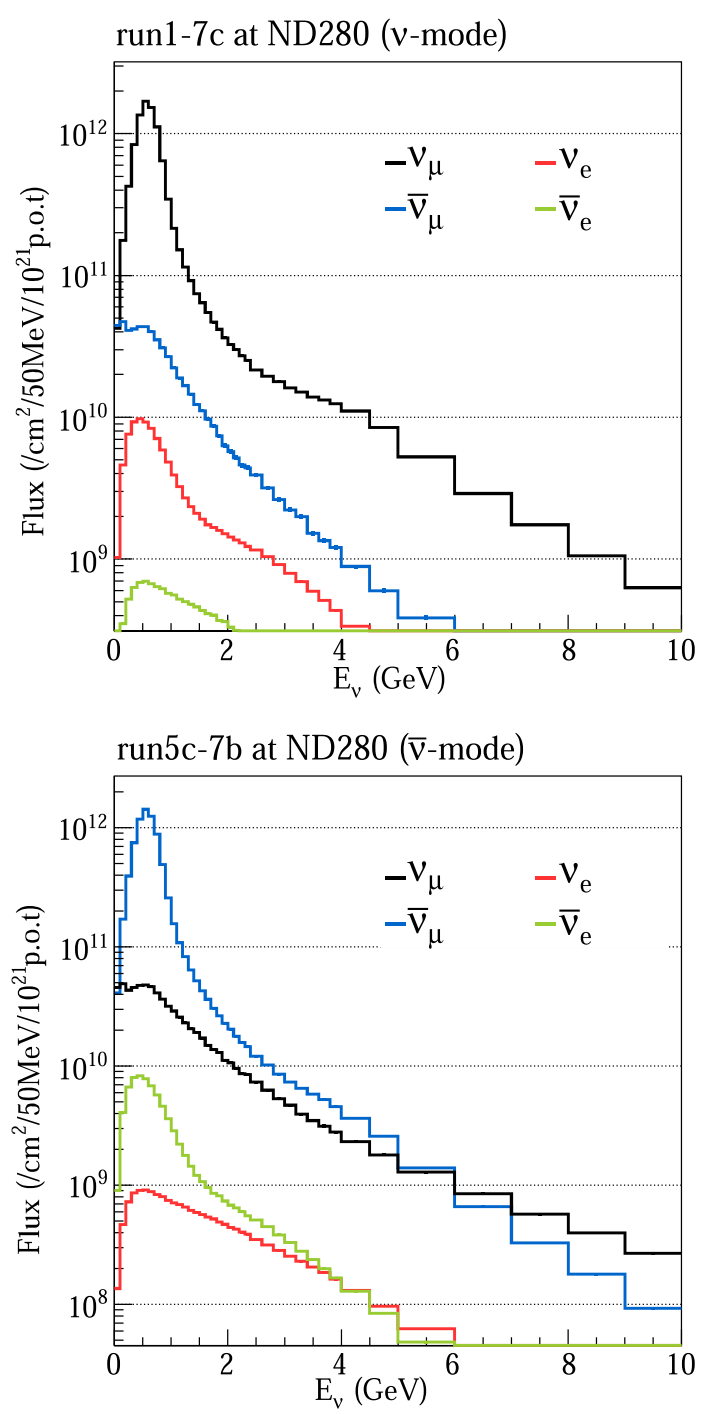

FIG. 1. The predominately neutrino FHC beam (Top) and predominately antineutrino RHC beam (Bottom) flux per PoT as a function of energy at the ND280 detector. The rates are separated by neutrino/antineutrino muon and electron type flavors. The peak values for the neutrino and the antineutrino flux rates are $1.7 \times 10^{12}$ and $1.4 \times 10^{12} / \mathrm{cm}^{2} / 50 \mathrm{MeV} / 10^{21} \mathrm{PoT}$, respectively.

neutrino beam direction, and the $+\mathrm{Y}$ direction is vertically upwards. Previous descriptions of analyses using the PØD have been published [23]. We describe additional details relevant for the analysis presented in this paper.

The PØD is shown in Fig. 2. This detector contains 40 scintillator module planes called PØDules. Each PØDule has 134 horizontal and 126 vertical triangular scintillator bars. A wavelength shifting fiber centered in each bar is readout on one end by a silicon photomultiplier. The PøD dimensions are $2298 \times 2468 \times 2350 \mathrm{~mm}^{3}-\mathrm{XYZ}$ - with a total mass of $\sim 1900 \mathrm{~kg}$ of water and $3570 \mathrm{~kg}$ of other materials (mainly scintillator with thin layers of high density polyethylene plastic and brass sheet). The target 


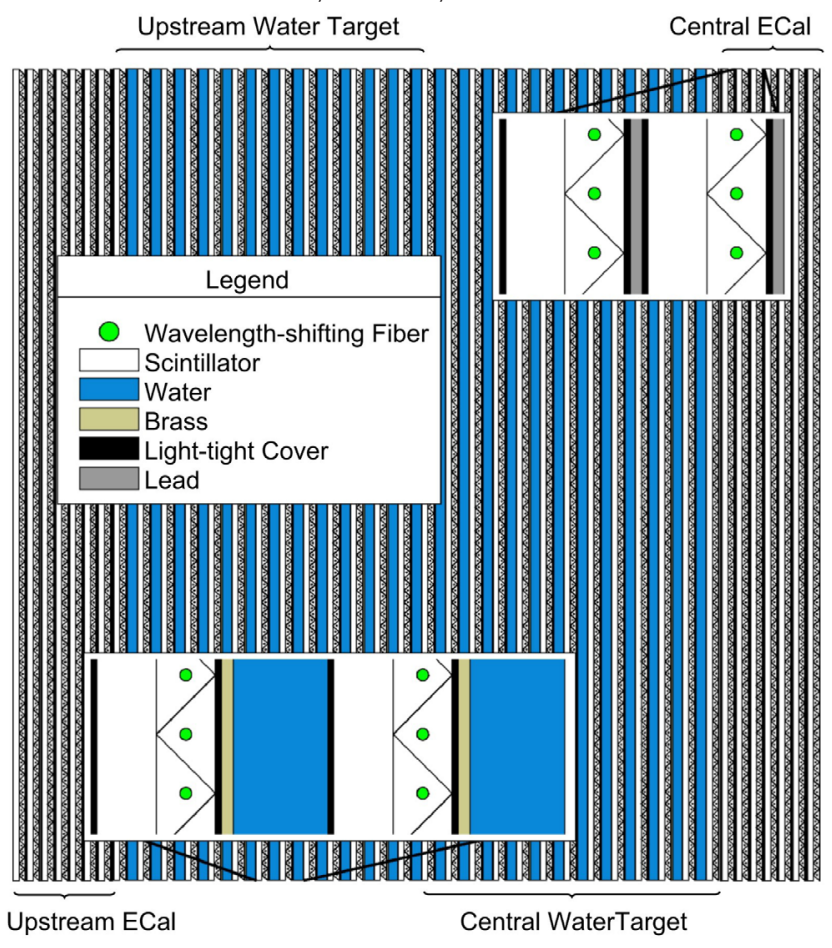

FIG. 2. Side view schematic diagram of the PØD detector. The white, zig-zag, and blue strip regions represent the vertical scintillator bars, the horizontal scintillator bars, and the water bag regions, respectively. The vertical and horizontal bars represent a X-Y module or $\mathrm{P} \emptyset$ Dule. The first and last groups of seven PØDules form the upstream and the central ECAL "super" modules and the middle 26 PØDules interleaved with the water bags are the water target region.

material mass is given in fractional amounts in Table I. These PØDules are formed into three major sections. The water target region, is the primary target in this analysis which has 26 PØDules interleaved with bags of water $2.8 \mathrm{~cm}$ thick and $1.3 \mathrm{~mm}$ brass sheets. The water bags are drainable to allow water target subtraction measurements. The two other regions (called upstream and central ECALs) are the upstream and downstream sections that each contain 7 PøDules and steel sheets clad with lead (4.9 radiation lengths).

TABLE I. Chemical element composition of PøD water target region by fraction of mass.

\begin{tabular}{lcc}
\hline \hline Element & Symbol & Fraction \\
\hline Hydrogen & $\mathrm{H}$ & $8.0 \%$ \\
Carbon & $\mathrm{C}$ & $45.0 \%$ \\
Oxygen & $\mathrm{O}$ & $29.9 \%$ \\
Copper & $\mathrm{Cu}$ & $14.3 \%$ \\
Chlorine & $\mathrm{Cl}$ & $1.1 \%$ \\
Titanium & $\mathrm{Ti}$ & $0.1 \%$ \\
Zinc & $\mathrm{Zn}$ & $1.6 \%$ \\
\hline \hline
\end{tabular}

The TPC1,2,3 detectors are three modules whose dimensions are each $1808 \times 2230 \times 852 \mathrm{~mm}^{3}-\mathrm{XYZ}-$ where each module contains a centered high voltage (Z-Y) cathode plane that splits the chamber into two sections where the charged particle track ionizations drift in the $\pm X$ directions. These are measured by $70 \mathrm{~mm}^{2}$ micromegas pads in the Z-Y plane. The fully contained ionized track path lengths are $72 \mathrm{~cm}$. A charged track will be measured with $\sim 0.7 \mathrm{~mm}$ resolution for drift distances $>10 \mathrm{~cm}$. The typical TPC momentum resolution is $\delta\left(p_{\perp}\right) / p_{\perp}=$ $0.08 p_{\perp}(\mathrm{GeV} / c)$. Analyses which use the TPC have been described in previous ND280 publications [20].

\section{ANALYSIS SAMPLES}

The studies reported here includes data logged with the FHC $\nu$ beam runs (October 2012 to February 2013) and the RHC $\bar{\nu}$ beam runs (May 2014 to June 2014).

\section{A. Data samples and detector configuration}

The total PoT exposure where all detector data quality checks were passed for the FHC runs was $16.24 \times 10^{19}$ and the corresponding total PoT exposure for the RHC runs was $4.30 \times 10^{19}$. These integrated rates corresponds to roughly $0.28 \times 10^{12}$ neutrinos and $0.06 \times 10^{12}$ antineutrinos per $\mathrm{cm}^{2}$ per $50 \mathrm{MeV}$ at $0.6 \mathrm{GeV}$. The data samples in this paper used the available neutrino and antineutrino beam data taken when the PØD target bags were filled with water.

\section{B. Monte Carlo simulation}

The analysis used simulated MC samples with different beam and detector configurations for each data-taking period. The simulations include the following:

(1) Secondary pions and kaons are produced in the graphite target and propagated through the magnetic horns into a helium filled pipe where they decay. Secondary neutrinos and antineutrinos are created and their fluxes and energy spectra are extrapolated to the near and far detectors.

(2) The neutrino and antineutrino interactions in the ND280 subdetectors were determined by the NEUT [24] MC generator that was used to calculate the interaction cross sections and the final state particle kinematics.

(3) The detector simulation uses GEANT to propagate the final state particles through the ND280 subdetectors.

\section{EVENT AND KINEMATIC SELECTION}

\section{A. Event selection}

The analysis selection uses reconstructed objects from both the PØD and TPC. Both subdetectors use independent reconstruction algorithms to generate objects from the raw data. The PØD uses a three-dimensional tracking algorithm 
to form tracks from individual hits in the scintillator bars. The TPC reconstruction uses a track in the Y-Z plane (nondrift plane) as a seed to search for hits in the downstream FGD to form a track object.

After independent reconstructions in the TPC and in the $\mathrm{P} \emptyset \mathrm{D}$, the analysis uses an algorithm to match a threedimensional PØD track ending near the most downstream edge of the PØD to a TPC track beginning near the most upstream edge of the TPC.

The event selection is the following:

(1) The first requirement is good data quality for the data run. After ND280 data is processed, the subdetectors are evaluated run by run for good timing with respect to the beam and checked to satisfy good detector calibrations. Events are used only if their run passed data quality checks. For each FHC (RHC) beam bunch there must be a negative (positive) TPC track that is identified within $\pm 70 \mathrm{~ns}$ around the nominal beam bunch time.

(2) A veto is applied to reject events whose vertex originated outside the fiducial region but had a secondary interaction inside the fiducial region. Also events with single tracks that are broken into two tracks by the track reconstruction are rejected. The event vertex is defined by the most upstream PøDule hit in the track. The vertex X-Y position is defined by the $X-Y$ triangular scintillator bars and the vertex $Z$ position of the $\mathrm{P} \emptyset \mathrm{Dule}$. The fiducial volume requires the vertex to be within $-836 \mathrm{~mm}<X<864 \mathrm{~mm}$ and $-871 \mathrm{~mm}<Y<869 \mathrm{~mm}$ and inside one of the middle 24 PØDules. The $X$ boundaries are $\sim 250 \mathrm{~mm}$ and the $\mathrm{Y}$ boundaries are $\sim 236 \mathrm{~mm}$ away from the ends of the $\mathrm{X}$ and $\mathrm{Y}$ scintillator bars, respectively.

(3) The vertex must be in the PØD water target fiducial volume. The charge is determined by the curvature of the TPC track. Of all TPC tracks meeting these criteria, the one with the highest reconstructed momentum at the start of the track is chosen to be the lepton candidate.

(4) The RHC mode selection has an additional requirement that the lepton track candidate is positively charged and has the highest momentum of all charged tracks in the bunch.

Due to the limited geometric acceptance of requiring a $\mathrm{CC}$ neutrino event vertex in the $\mathrm{P} \emptyset \mathrm{D}$ with its muon track detected in the TPC, this analysis is inherently not sensitive to the entire muon kinematic phase space. For this reason, we define a restricted phase space, described in the next subsection, that will cover the part of the kinematic phase space where we have good acceptance. Events that are reconstructed to have muon kinematics outside of the restricted phase space will be rejected. For the FHC mode selection, 19,259 events are selected in data. The number of selected events in the corresponding MC sample, scaled to the same data PoT exposure is 19,566. In RHC mode, 1,869 events are selected in data and the scaled MC sample has 1,953 events. The muon $p$ and $\theta$ distributions for data events with MC predictions are shown for both modes in Figs. 3 (left and middle) and 4 (left and middle), respectively. The plots include colored stacked histograms of MC interaction types to graphically display the composition of the selected events.

The fractional NEUT interaction types for the FHC and the RHC beam modes are given in Table II for the selected events described in Sec. IV. The MC channels defined [25] at the initial interaction vertex according to NEUT are CCQE (QE), 2p2h, CC with 1 charged pion (1Pi), CC with $>1$ charged pion (NPi), CC with $\mathrm{K}$ or $\eta$ meson (Meson), deep inelastic scattering (DIS), neutral current (NC), neutrino or antineutrino interaction $(\nu$ or $\bar{\nu})$, and events whose true vertex position was outside the fiducial volume (outFV) region of the PØD. The resulting selected events, according to the $\mathrm{MC}$ simulation, are predominately $\mathrm{CCQE}$, followed by CC events with 1 pion. Due to a substantial $\nu_{\mu}$
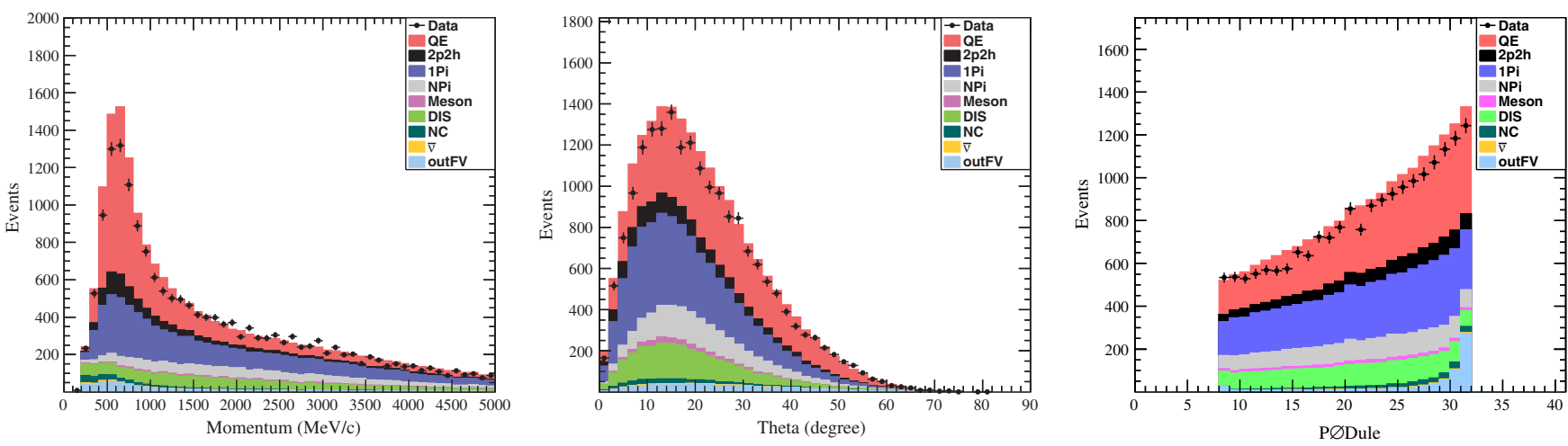

FIG. 3. FHC beam CCINC $\nu_{\mu}$ event candidate distributions of the $\mu^{-}$momentum in $\mathrm{MeV} / \mathrm{c}$ (left), the muon $\theta_{\mu}$ angle in degrees (middle), and interaction vertex position by PØDule (right). Note backgrounds in the CCINC sample are the NC (dark green), $\bar{\nu}_{\mu}$ induced events (yellow) and the out of fiducial volume events (light blue). There are negligible $\bar{\nu}_{\mu}$ backgrounds (yellow) in the FHC sample. 

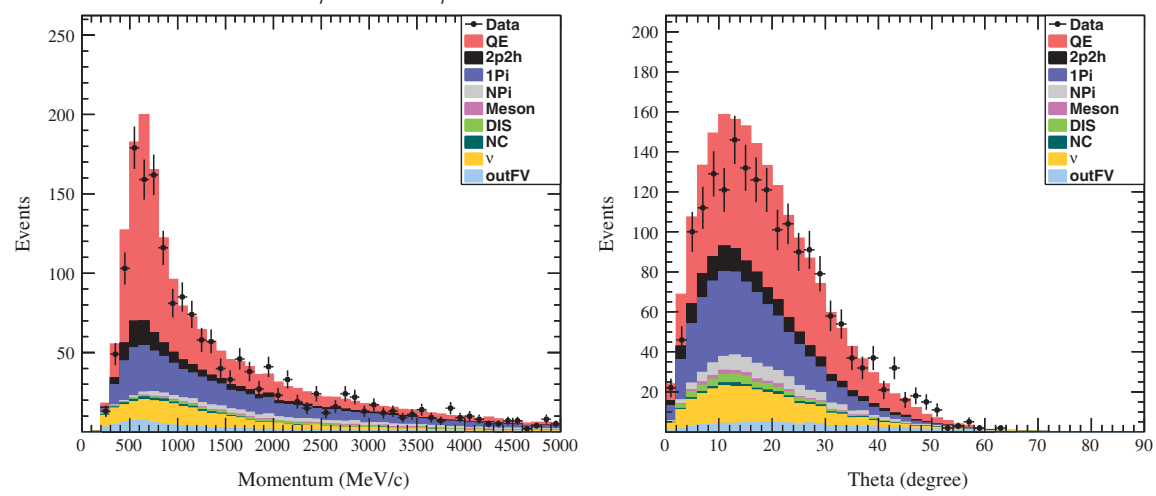

PHYSICAL REVIEW D 96, 052001 (2017)

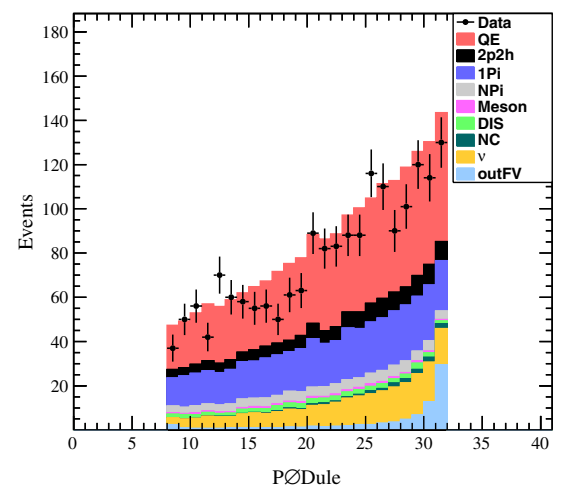

FIG. 4. RHC beam CCINC $\bar{\nu}_{\mu}$ event candidate distributions of the $\mu^{+}$momentum in MeV/c (left), the muon $\theta_{\mu}$ angle in degrees (middle), and interaction vertex position by PØDule (right). Note backgrounds in the CCINC sample are the NC (dark green), $\nu_{\mu}$ induced events (yellow) and the out of fiducial volume events (light blue). The $\nu_{\mu}$ backgrounds in the RHC beam sample are much larger than the analogous $\bar{\nu}_{\mu}$ backgrounds in the FHC beam sample.

flux contamination in the RHC beam and a large $\nu_{\mu}$ cross section, the $\bar{\nu}_{\mu}$ candidate sample has a larger background fraction (see yellow band in Fig. 4) compared to the $\bar{\nu}_{\mu}$ background events in the FHC beam sample. The $\nu_{\mu}$ in the RHC beam flux is seen in Fig. 1 (Bottom). The outFV backgrounds are roughly the same fraction in both $\mathrm{FHC}$ and RHC beam samples. The selection produces a CCINC $\nu_{\mu}$ candidate event sample that is $94.8 \%$ pure and a CCINC $\bar{\nu}_{\mu}$ candidate event sample that is $83.0 \%$ pure. The outFV backgrounds cluster in the light blue bands in Figs. 3 (right) and 4 (right) in the downstream PØDules. These backgrounds are events whose vertices are outside and downstream of the fiducial volume but with an interaction that has a backwards going track that enters the fiducial volume.

Additional checks between the data and MC event selections were performed by comparing the event rates of vertices by detector PØDule between data and

TABLE II. The fractional distributions of true MC interactions for selected events defined at the initial interaction vertex according to the NEUT generator for the FHC beam (left) and RHC beam (right) modes. See text for descriptions of each MC channel.

\begin{tabular}{lrcr}
\hline \hline & FHC beam & \multicolumn{2}{c}{ RHC beam } \\
Mode & Fraction & Mode & Fraction \\
\hline QE & $37.83 \%$ & QE & $47.27 \%$ \\
$2 \mathrm{p} 2 \mathrm{~h}$ & $3.30 \%$ & $2 \mathrm{p} 2 \mathrm{~h}$ & $3.19 \%$ \\
$1 \mathrm{Pi}$ & $29.73 \%$ & $1 \mathrm{Pi}$ & $24.14 \%$ \\
$\mathrm{NPi}$ & $11.01 \%$ & $\mathrm{NPi}$ & $5.05 \%$ \\
Meson & $1.71 \%$ & Meson & $1.04 \%$ \\
DIS & $11.27 \%$ & $\mathrm{DIS}$ & $2.32 \%$ \\
NC & $1.50 \%$ & $\mathrm{NC}$ & $0.99 \%$ \\
$\bar{\nu}_{\mu}$ & $0.33 \%$ & $\nu_{\mu}$ & $11.93 \%$ \\
outFV & $3.32 \%$ & outFV & $4.05 \%$ \\
\hline \hline
\end{tabular}

normalized selected $\mathrm{MC}$ events. The event rates by PØDule are shown for $\nu_{\mu}$ and $\bar{\nu}_{\mu}$ in Figs. 3 (right) and 4 (right), respectively. There is very good agreement within statistics between the data and MC distributions, except the momentum distribution in the FHC beam sample where the data are 1-2 sigma below the MC predictions near $0.6 \mathrm{GeV} / \mathrm{c}$. The efficiency for the $\nu_{\mu}$ and $\bar{\nu}_{\mu}$ events varies as a function of $\mathrm{P} \emptyset$ Dule. Since the event selection requires a vertex in a PØDule with a muon track reconstructed in the TPC, the downstream PØDules have a higher efficiency than the upstream PØDules. The events with vertices in the more upstream $P \emptyset$ Dule have smaller angular acceptance for a muon track to pass through the TPC and the muon track will incur more energy loss since it must pass through more PØDules to reach the TPC where it must be reconstructed. The $\nu$ event selection efficiency in Fig. 3 (right) from upstream to downstream PØDule varies from 37\% to 57\% whereas the $\bar{\nu}_{\mu}$ event selection efficiency Fig. 4 (right) varies from $39 \%$ to $68 \%$.

\section{B. Kinematic selection}

The selected events for the RHC (FHC) samples require a vertex in the $\mathrm{P} \emptyset \mathrm{D}$ and a $\mu^{+}\left(\mu^{-}\right)$reconstructed track in the TPC detector. This limits or restricts the available kinematic phase space of the CCINC events such that certain kinematic regions are not measured. These unmeasured regions in the laboratory frame have low muon momentum $p_{\mu}<500 \mathrm{MeV} / \mathrm{c}$ or large muon polar angles $\theta_{\mu}>32^{\circ}$.

These kinematic boundaries are displayed in Figs. 5 and 6 left (right) where the $\theta_{\mu}$ versus $p_{\mu}$ two-dimensional plots are shown for the RHC (FHC) samples. In Fig. 5 left (right) are the generated $\mathrm{MC}$ full acceptance CCINC events for the RHC (FHC) samples. The $\nu_{\mu}$ mode has more events with larger $\theta_{\mu}$ polar angles since the $\mu^{-}$angular distribution is more isotropic than the $\mu^{+}$in the $\bar{\nu}_{\mu}$ mode whose muon 

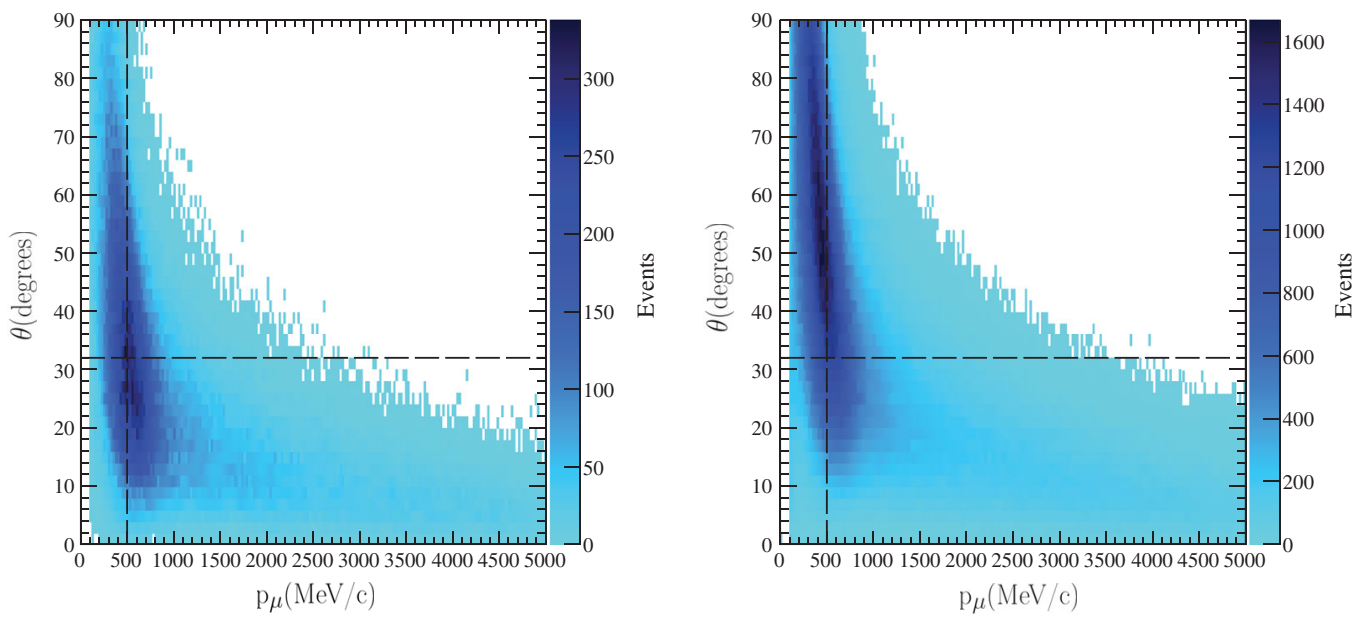

FIG. 5. Left (right) two-dimensional plots of $\theta_{\mu}$ versus $p_{\mu}$ for RHC (FHC) beam events of $\mu^{+}\left(\mu^{-}\right)$tracks using MC generated CCINC with full acceptance. The vertical and horizontal solid lines correspond to $\theta_{\mu}=32^{\circ}$ and $p_{\mu}=500 \mathrm{MeV} / \mathrm{c}$, respectively.

tracks are more forward. In Fig. 6 left (right) are the generated MC CCINC events that have a PØD vertex and a $\mu^{+}\left(\mu^{-}\right)$track reconstructed in the TPC for the RHC (FHC) samples. The regions below horizontal lines where $\theta_{\mu}<32^{\circ}$ and right of the vertical dash lines where $p_{\mu}>$ $500 \mathrm{MeV} / \mathrm{c}$ are detector regions that have nonzero acceptance and reconstructed events for both the FHC and the RHC samples. Hence, we use these two kinematic restrictions in the cross-section measurements. The resulting reconstructed restricted phase space selection in the $\nu_{\mu}$ mode has 14,398 data events and a corresponding MC sample, scaled to the same data PoT exposure, contains 15,284 events. In the $\bar{\nu}_{\mu}$ mode, 1,461 data events are selected and a scaled MC sample has 1,634 events. From a study of MC truth selected events, this restricted phase space selection changed the mean value of neutrino

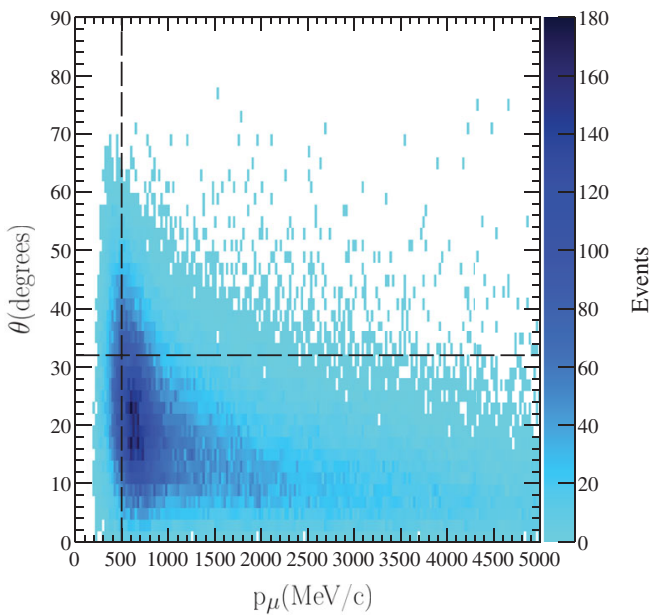

energies below $2 \mathrm{GeV}$ in the FHC sample from $0.83 \mathrm{GeV}$ (unrestricted) to $1.14 \mathrm{GeV}$ (restricted) and in the RHC sample from $0.84 \mathrm{GeV}$ (unrestricted) to $1.08 \mathrm{GeV}$ (restricted). In addition, the $\nu_{\mu}$ and $\bar{\nu}_{\mu}$ MC samples contained $2.19 \%$ and $1.33 \%$ events, respectively, whose true kinematic value was outside the restricted phase space region, but its reconstructed value migrated to be inside the restricted phase space region. These events are kinematic backgrounds that originated from the same physics process.

\section{ANALYSIS METHODS}

The number of neutrino interactions in the fiducial volume of the $\mathrm{P} \emptyset \mathrm{D}, N_{\text {signal }}$, can be expressed as the product of the signal cross section per target, $\sigma$, the number of

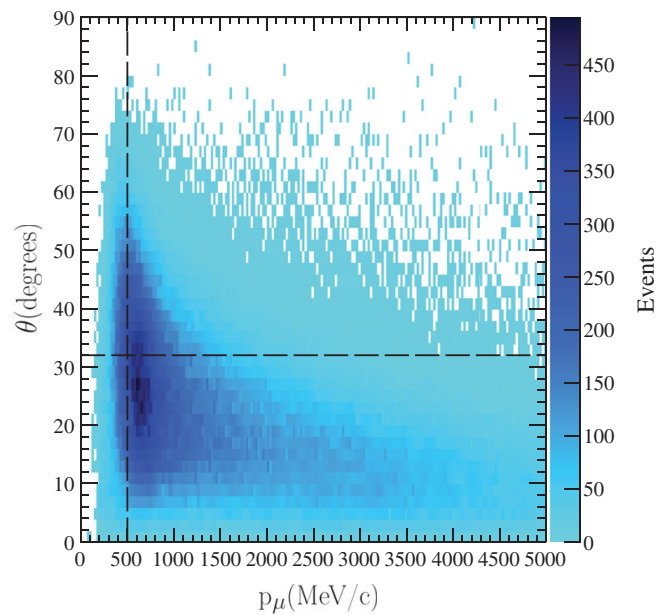

FIG. 6. Left (right) two-dimensional plots of $\theta_{\mu}$ versus $p_{\mu}$ for RHC (FHC) beam events of $\mu^{+}\left(\mu^{-}\right)$tracks using MC generated CCINC that have a reconstructed PØD vertex and TPC muon track. The vertical and horizontal solid lines correspond to $\theta_{\mu}=32^{\circ}$ and $p_{\mu}=500 \mathrm{MeV} / \mathrm{c}$, respectively. The restricted phase space cut selection applies to events inside the lower right rectangular region defined by the dashed lines. 
targets, $N_{\text {targets, }}$, and the integrated flux, $\Phi$, of incident neutrinos per unit area, as

$$
N_{\text {signal }}=\sigma N_{\text {targets }} \Phi
$$

Hence, the cross section becomes

$$
\sigma=\frac{N_{\text {signal }}}{\Phi N_{\text {targets }}} .
$$

Using our event selection on data, we obtain a candidate signal event sample in our fiducial volume. This process is not $100 \%$ efficient and also some nonsignal (background) events are included. To account for this, the MC simulation is used to estimate in our sample the number of background events and the number of signal events. The backgrounds from the FHC (RHC) beam samples include non-CCINC events from the neutrino (antineutrino) beam as well as events created from the antineutrino (neutrino) flux. In addition, the MC simulation generates total number of signal events that were produced. If the rate of restricted phase space selected data events is $N_{\text {selected }}^{\text {data }}$ and the predicted number of selected background events is $B^{\mathrm{MC}}$, the observed number of signal candidates in our fiducial volume is

$$
N_{\text {selected signal }}=N_{\text {selected }}^{\text {data }}-B^{\mathrm{MC}} \text {, }
$$

which include migration events. Next we redefine the selection efficiency $\epsilon$ as

$$
\epsilon=\frac{N_{\text {selected signal }}^{\mathrm{MC}}}{N_{\text {generated signal }}^{\mathrm{MC}}} .
$$

where the $N_{\text {selected signal }}^{\mathrm{MC}}$ is the number of signal candidates whose reconstructed kinematics are in the restricted phase space and $N_{\text {generated signal }}^{\mathrm{MC}}$ is the total number of generated signal events whose true kinematics are in the restricted phase space. We note that $N_{\text {selected signal }}^{\mathrm{MC}}$ includes a small fraction of migration events as described at the end of Sec. IV. B. With these definitions, the restricted phase space signal event rate is

$$
N_{\text {signal }}=\frac{N_{\text {selected }}^{\text {data }}-B^{\mathrm{MC}}}{\epsilon} .
$$

In Eq. (5), the numerator is the number of signal candidates whose reconstructed kinematics are in the restricted phase space, and this is combined with the denominator $\epsilon$ from Eq. (4) to give the proper estimate of $N_{\text {signal }}$ that represents the number of signal events whose kinematics are in the true restricted phase space. The neutrino cross section is

$$
\sigma\left(\nu_{\mu}\right)=\frac{N_{\text {selected }}^{\text {data }}-B^{\mathrm{MC}}}{\epsilon N_{\text {targets }} \Phi} .
$$

In addition to the cross sections given above, the measured ratio of cross sections $R(\nu, \bar{\nu})$ and rates $r(\nu, \bar{\nu})$ are defined as

$$
R(\nu, \bar{\nu}) \equiv \frac{\sigma\left(\bar{\nu}_{\mu}\right)}{\sigma\left(\nu_{\mu}\right)}=\frac{\bar{N}_{\text {selected }}^{\text {data }}-\bar{B}^{\mathrm{MC}}}{N_{\text {selected }}^{\text {dat }}-B^{\mathrm{MC}}} \times \frac{\bar{\epsilon}}{\epsilon} \times \frac{\Phi}{\bar{\Phi}}
$$

and

$$
r(\nu, \bar{\nu}) \equiv \frac{n\left(\bar{\nu}_{\mu}\right)}{n\left(\nu_{\mu}\right)}=\frac{\bar{N}_{\text {selected }}^{\text {data }}-\bar{B}^{\mathrm{MC}}}{N_{\text {selected }}^{\text {data }}-B^{\mathrm{MC}}} \times \frac{\bar{\epsilon}}{\epsilon} .
$$

The overlined quantities are obtained from the antineutrino selections as described above and those without overlines represent the neutrino mode selection. Finally, other observables are introduced and defined; the sum $\Sigma(\nu, \bar{\nu})$, difference $\Delta(\nu, \bar{\nu})$, and asymmetry $A(\nu, \bar{\nu})$ formed from the $\nu_{\mu}$ and $\bar{\nu}_{\mu}$ cross sections, as

$$
\begin{gathered}
\Sigma(\nu, \bar{\nu}) \equiv \sigma\left(\nu_{\mu}\right)+\sigma\left(\bar{\nu}_{\mu}\right), \\
\Delta(\nu, \bar{\nu}) \equiv \sigma\left(\nu_{\mu}\right)-\sigma\left(\bar{\nu}_{\mu}\right)
\end{gathered}
$$

and

$$
A(\nu, \bar{\nu}) \equiv \frac{\sigma\left(\nu_{\mu}\right)-\sigma\left(\bar{\nu}_{\mu}\right)}{\sigma\left(\nu_{\mu}\right)+\sigma\left(\bar{\nu}_{\mu}\right)} .
$$

\section{CROSS SECTION AND RATIO SYSTEMATIC ERRORS}

The systematic errors on cross sections and ratios of cross sections in this analysis are due to uncertainties on the number of selected background events, the incident neutrino flux, the number of targets in the detector, and the selection efficiencies. The sources of systematic uncertainties can be categorized into three groups: beam flux prediction, neutrino and antineutrino interaction models and detector response. The largest source of uncertainty is due to the beam flux.

\section{A. Beam flux uncertainty}

The beam flux uncertainty sources can be separated into two categories: uncertainties of the hadronic interactions, in the graphite target and reinteractions in the horn, and T2K beam line inaccuracies.

The beam flux uncertainty is dominated by the uncertainty on the modeling of the hadron interactions, including uncertainties on the total proton-nucleus production cross section, pion and kaon multiplicities, and secondary nucleon production. 
TABLE III. Summary table for one standard deviation errors due to beam flux uncertainties (fractionl errors in \%).

\begin{tabular}{lccccc}
\hline \hline$\sigma(\bar{\nu})$ & $\sigma(\nu)$ & $R(\nu, \bar{\nu})$ & $A(\nu, \bar{\nu})$ & $\Sigma(\nu, \bar{\nu})$ & $\Delta(\nu, \bar{\nu})$ \\
\hline \pm 9.37 & \pm 9.14 & \pm 3.58 & \pm 3.35 & \pm 9.17 & \pm 9.42 \\
\hline \hline
\end{tabular}

The hadronic interactions in the target where the primary proton beam first interacts and produces the majority of the secondary pions is simulated by the FLUKA2011 package which creates MC neutrino and antineutrino flux samples. Uncertainties on the proton beam properties, horn current, hadron production model and alignment are taken into account to produce an energy-dependent systematic uncertainty on the neutrino flux. These uncertainties are propagated to the $\mathrm{T} 2 \mathrm{~K}$ neutrino beam flux prediction by reweighting MC flux samples. Systematic uncertainties on the neutrino flux predictions coming from the NA61/ SHINE hadron production measurements are included. Those uncertainties were estimated by varying track selection and identification criteria as well as the parameters used to calculate needed corrections, to account for example for decays of strange particles like Lambdas, which produce additional pions and protons, that can mimic our signal in the NA61/SHINE detector. Detailed review of the sources of systematics errors of hadron production data of NA61/SHINE results needed for T2K may be found elsewhere [19].

The flux smearing is done using toy $\mathrm{MC}$ data sets that are based on the FHC and RHC beam flux uncertainty covariance matrices. The resulting $\pm 1 \sigma$ change in the cross section is taken as the systematic error associated with the beam flux. These uncertainties on individual cross sections lead to 9\% errors whereas the errors on the ratio are $4 \%$ due to correlated neutrino and antineutrino flux covariance errors. Table III summarizes the systematic errors due to the beam flux uncertainties on the cross sections and combinations of cross sections. These results have been cross checked with analytic calculations. The fractional errors on ratios have smaller errors due to cancellations of correlated errors between the neutrino and antineutrino modes.

\section{B. Interaction model uncertainty}

The interaction model uncertainties were calculated by a data-driven method [26] where the NEUT predictions were compared to external neutrino-nucleus data in the energy region relevant for T2K. Some of the NEUT model parameters are fitted and assigned mean and $1 \sigma$ error values that allow for differences between NEUT and the external data.

The CCQE model in NEUT is based on the LlewellynSmith neutrino-nucleon scattering model [27] with a dipole axial form factor and the BBBA05 vector form factors [28]. The NEUT generator uses the Smith-Moniz RFG model [29] and includes an implementation of both the random phase approximation (RPA) correction [30] and the $2 \mathrm{p} 2 \mathrm{~h}$ Nieves model [30]. The NEUT resonant pion production is based on the Rein-Sehgal model [31] with updated form factors from Ref. [32]. The DIS model used in NEUT includes both the structure function from Ref. [33] and the Bodek-Yang correction [34]. The NEUT MC generator includes various model parameters to describe the different models, uncertainties and approximations. The axial mass $M_{A}^{\mathrm{QE}}$ was set to $1.21 \mathrm{GeV} / c^{2}$ based on the SuperKamiokande atmospheric data and the $\mathrm{K} 2 \mathrm{~K}$ data. The $1 \sigma$ error on $M_{A}^{\mathrm{QE}}$ was set to $0.41 \mathrm{GeV} / c^{2}$. The large uncertainty on this parameter is due to the disagreements between recent experimental measurements and bubble chamber results [35]. The Fermi gas momentum parameter $\left(p_{F}\right)$ values and their errors are set to $223 \mathrm{MeV} / \mathrm{c}$ and $225 \mathrm{MeV} / \mathrm{c}$ for carbon and oxygen, respectively, with both errors set to $\pm 12.7 \mathrm{MeV} / \mathrm{c}$. The Fermi gas binding energy $\left(E_{B}\right)$ parameter was set to $25 \mathrm{MeV}$ and $27 \mathrm{MeV}$ for carbon and oxygen, respectively, with both errors set to $\pm 9 \mathrm{MeV}$. The Nieves model $2 \mathrm{p} 2 \mathrm{~h}$ normalization to $1 \pm 1$ for both carbon and oxygen, the resonant pion production model in NEUT used the Graczyk and Sobczyk form factors $C_{5}^{A}(0)$ and the $I=\frac{1}{2}$ background scale were set to $1.01 \pm 0.12$ and $1.20 \pm 0.20$, respectively. The nominal axial mass $M_{A}^{\mathrm{RES}}$ was set to $0.95 \pm 0.15 \mathrm{GeV} / \mathrm{c}^{2}$. Additional uncertainties are $\nu_{e} / \nu_{\mu}$ cross section factor that was set to $1.00 \pm 0.02$. Both CC and NC coherent uncertainties based on the ReinSehgal model were set to $1 \pm 1$ and $1.0 \pm 0.3$, respectively. Moreover, for $\mathrm{CC}$ and $\mathrm{NC}$ interactions, additional scale factors were set to $0.0 \pm 0.4$ and $1.0 \pm 0.3$, respectively. In addition the $\mathrm{CC}$ other is an energy dependent factor [20] and the $\mathrm{NC}$ other is a normalization factor. The $\pi$ final state interaction (FSI) uncertainties are tuned to a pion-nucleus scattering data, and other smaller corrections were included [26].

Variation of model parameters within their errors $( \pm 1 \sigma)$ was used to estimate their effect on the final observables in order to determine final measurement uncertainties. A summary of the parameters and their effects on the overall normalization are shown in Table IV.

\section{Detector response uncertainty}

The detector response uncertainty studies used data samples supported with MC samples and measurements of the target weight. The three dominant detector response systematic uncertainties are caused by the fiducial volume boundaries, the sand/rock muon interactions and the mass of the target in the fiducial volume. There were small uncertainties from reconstruction and charge misidentification from the TPC measurements. All the sources of detector response errors considered in the analysis are given in Table V.

The fiducial volume systematics were estimated by varying its boundaries. The sand/rock muon interactions 
TABLE IV. Summary table for physics model uncertainties for restricted phase space measurements (fractional errors in \%).

\begin{tabular}{|c|c|c|c|c|c|c|}
\hline Parameter & $\sigma(\bar{\nu})$ & $\sigma(\nu)$ & $R(\nu, \bar{\nu})$ & $A(\nu, \bar{\nu})$ & $\Sigma(\nu, \bar{\nu})$ & $\Delta(\nu, \bar{\nu})$ \\
\hline$\overline{M_{A}^{\mathrm{QE}}}$ & \pm 0.51 & \pm 0.14 & \pm 0.37 & \pm 0.32 & \pm 0.24 & \pm 0.08 \\
\hline$p_{F}{ }^{A}\left({ }^{12} \mathrm{C}\right)$ & \pm 0.01 & \pm 0.02 & \pm 0.01 & \pm 0.01 & \pm 0.02 & \pm 0.02 \\
\hline$p_{F}\left({ }^{16} \mathrm{O}\right)$ & 0 & \pm 0.01 & 0 & 0 & \pm 0.01 & \pm 0.01 \\
\hline MEC norm $\left({ }^{12} \mathrm{C}\right)$ & \pm 0.30 & \pm 0.44 & \pm 0.14 & \pm 0.12 & \pm 0.40 & \pm 0.52 \\
\hline MEC norm $\left({ }^{16} \mathrm{O}\right)$ & \pm 0.18 & \pm 0.24 & \pm 0.06 & \pm 0.05 & \pm 0.22 & \pm 0.27 \\
\hline$E_{B}\left({ }^{12} \mathrm{C}\right)$ & \pm 0.01 & \pm 0.01 & \pm 0.02 & \pm 0.02 & 0 & \pm 0.02 \\
\hline$E_{B}\left({ }^{16} \mathrm{O}\right)$ & \pm 0.01 & \pm 0.01 & \pm 0.02 & \pm 0.02 & 0 & \pm 0.02 \\
\hline$C_{5}^{A}(0)$ & \pm 0.70 & \pm 0.46 & \pm 0.24 & \pm 0.21 & \pm 0.53 & \pm 0.32 \\
\hline$M_{A}^{1 \pi}$ & \pm 0.99 & \pm 0.28 & \pm 0.75 & \pm 0.65 & \pm 0.44 & \pm 0.21 \\
\hline$I=\frac{1}{2} \mathrm{Bkg}$ & \pm 0.29 & \pm 0.21 & \pm 0.08 & \pm 0.07 & \pm 0.23 & \pm 0.17 \\
\hline$\nu_{e} / \nu_{\mu}^{2}$ & \pm 0.02 & 0 & \pm 0.01 & \pm 0.01 & \pm 0.01 & 0 \\
\hline CC Other shape & \pm 0.65 & \pm 0.70 & \pm 0.06 & \pm 0.79 & \pm 0.06 & \pm 0.75 \\
\hline CC Coherent & \pm 0.01 & \pm 0.01 & 0 & \pm 0.05 & \pm 0.69 & \pm 0.73 \\
\hline NC Coherent & 0 & 0 & 0 & 0 & 0 & 0 \\
\hline NC Other & \pm 1.28 & \pm 0.39 & \pm 0.89 & \pm 0.77 & \pm 0.63 & \pm 0.14 \\
\hline$\pi$ FSI & \pm 0.16 & \pm 0.19 & \pm 0.11 & \pm 0.09 & \pm 0.18 & \pm 0.23 \\
\hline MEC norm Other & \pm 0.08 & \pm 0.15 & \pm 0.07 & \pm 0.20 & \pm 0.13 & \pm 0.20 \\
\hline Total & \pm 2.13 & \pm 1.16 & \pm 1.56 & \pm 1.36 & \pm 1.31 & \pm 1.32 \\
\hline
\end{tabular}

TABLE V. Summary table for detector response uncertainties (fractional errors in \%).

\begin{tabular}{|c|c|c|c|c|c|c|}
\hline Parameter & $\sigma(\bar{\nu})$ & $\sigma(\nu)$ & $R(\nu, \bar{\nu})$ & $A(\nu, \bar{\nu})$ & $\Sigma(\nu, \bar{\nu})$ & $\Delta(\nu, \bar{\nu})$ \\
\hline TPC tracking efficiency & \pm 0.37 & \pm 0.32 & \pm 0.04 & \pm 0.04 & \pm 0.34 & \pm 0.29 \\
\hline Charge misidentification & \pm 0.37 & \pm 0.32 & \pm 0.04 & \pm 0.04 & \pm 0.34 & \pm 0.29 \\
\hline Sand/Rock muon interference & \pm 1.45 & \pm 2.20 & \pm 0.74 & \pm 0.70 & \pm 1.99 & \pm 2.70 \\
\hline Fiducial mass & \pm 0.96 & \pm 0.96 & 0 & 0 & \pm 1.36 & \pm 1.36 \\
\hline Fiducial volume boundaries & \pm 0.13 & \pm 0.97 & \pm 0.83 & \pm 1.39 & \pm 0.77 & \pm 0.74 \\
\hline Total & \pm 1.82 & \pm 2.63 & \pm 1.11 & \pm 1.02 & \pm 2.58 & \pm 3.35 \\
\hline
\end{tabular}

occurring upstream and in the surrounding ND280 volume could create tracks passing through the PØD and TPC detectors, mimicking a CCINC event. Another source of detector systematics was the mass of the target in the fiducial volume. The uncertainty due to the fiducial mass was conservatively estimated to be $0.96 \%$ from the measured mass of the detector material during construction and the water mass measured during filling the water bags.

\section{RESULTS}

\section{A. Cross sections and ratios}

The flux averaged cross section and ratio values measured in the FHC and RHC samples are extracted from the flux, the number of targets, $\mathrm{MC}$ efficiencies and $\mathrm{MC}$ background estimates. The input parameters are given in Table VI, and the results for the restricted (full) phase space selections are given in Tables VII (VIII). The systematic errors in Table VII are determined by adding in quadrature the errors in Tables III, IV and V. For example, the fractional $\mathrm{R}$ error, taken from the three tables, is $4 \%$ and this yields 0.015 for the absolute systematic $\mathrm{R}$ error in Table VII.

In Table VI, for the restricted phase space results, the input parameters include the $\nu_{\mu}\left(\bar{\nu}_{\mu}\right)$ fluxes normalized to PoT in the FHC(RHC) samples. The number of nucleon targets is given for both the data and MC which slightly differed. The number of reconstructed MC events is given

TABLE VI. Tabulation of flux, targets, and data/MC events used in the cross section calculations. The data corrected values are background subtracted and divided by the MC efficiency.

\begin{tabular}{lccc}
\hline \hline Inputs for cross sections & Units & RHC $\bar{\nu}$ mode & FHC $\nu$ mode \\
\hline Integrated flux & {$\left[\mathrm{cm}^{2} / 10^{21}\right.$ PoT] } & $1.477 \times 10^{13}$ & $1.823 \times 10^{13}$ \\
Number of targets (data) & {$[$ Nucleons] } & $3.147 \times 10^{30}$ & $3.147 \times 10^{30}$ \\
Number of targets (MC) & {$[$ Nucleons] } & $3.119 \times 10^{30}$ & $3.119 \times 10^{30}$ \\
Number of data/MC events (restricted PS) & {$[$ Events] } & $1,498 / 1,634$ & $14,398 / 15,284$ \\
Data corrected (restricted PS) & {$\left[\right.$ Events/10 $/ 10^{21}$ PoT] } & $41,821 \pm 1,334$ & $138,576 \pm 1,249$ \\
\hline \hline
\end{tabular}


TABLE VII. Restricted phase space cross section and ratio final results.

\begin{tabular}{lccc}
\hline \hline & \multicolumn{3}{c}{ Cross sections $\left[\times 10^{-39} \mathrm{~cm}^{2} /\right.$ nucleon $]$} \\
\hline$\sigma(\bar{\nu})$ & 0.900 & \pm 0.029 (stat.) & \pm 0.088 (syst.) \\
$\sigma(\nu)$ & 2.41 & \pm 0.022 (stat.) & \pm 0.231 (syst.) \\
$\Delta(\nu, \bar{\nu})$ & 1.512 & \pm 0.036 (stat.) & \pm 0.152 (syst.) \\
$\Sigma(\nu, \bar{\nu})$ & 3.311 & \pm 0.036 (stat.) & \pm 0.318 (syst.) \\
& \multicolumn{3}{|}{ Ratios } \\
$R(\nu, \bar{\nu})$ & 0.373 & \pm 0.012 (stat.) & \pm 0.015 (syst.) \\
$A(\nu, \bar{\nu})$ & 0.457 & \pm 0.012 (stat.) & \pm 0.017 (syst.) \\
\hline \hline
\end{tabular}

scaled to the equivalent data PoT. The data/MC generated corrected events are defined as the reconstructed data/MC generated events, minus the $\mathrm{MC}$ background and divided by the MC CCINC efficiencies.

In Table VIII, the full phase space results are extrapolated by scaling the restricted values in Table VI by the ratio of the total to restricted cross sections as predicted by the NEUT MC generator. The single errors combine the statistical and systematic errors, which included model uncertainties on the assumed values of $M_{A}^{\mathrm{QE}}$ and the $2 \mathrm{p} 2 \mathrm{~h}$ $C^{12}$ and $O^{16}$ parameters in the scaling factor. The errors on the $\nu_{\mu}$ and $\bar{\nu}_{\mu}$ cross sections due to these parameter uncertainties were assumed to be totally uncorrelated leading to a conservative estimate of the systematic errors on the full phase space ratio of cross sections.

The cross section calculations use Eq. (6), and the ratio $R(\nu, \bar{\nu})$ is obtained from Eq. (7), where we note the number of targets drops out. We find $\approx 10 \%$ systematic cross section errors whereas the ratio of cross sections $R(\nu, \bar{\nu})$ error has a factor $\times 2$ smaller values of $4.0 \%$ errors for the restricted phase space. These systematic errors are mainly due to the flux uncertainties on the flux prediction which have strong correlations between neutrino and antineutrino fluxes which largely cancel in the ratio. The flux predictions for neutrino mode and antineutrino mode are correlated through measurements that are used as inputs to the flux calculation. These measurements include the proton beam current measurement, the measurement of the primary proton interaction rate by NA61/SHINE, and the measurement of secondary particle interaction rates by other hadron interaction experiments. The measured ratio of rates $r(\nu, \bar{\nu})$ given in Eq. (8) represents the ratio of $\nu_{\mu}$ and $\bar{\nu}_{\mu}$ event rates which depends on the integrated FHC and RHC flux and so

TABLE VIII. Full phase space cross sections and ratio results extrapolated from restricted phase space measurements.

\begin{tabular}{lcc}
\hline \hline & Cross sections $\left[\times 10^{-39} \mathrm{~cm}^{2} /\right.$ nucleon $]$ \\
\hline$\sigma(\bar{\nu})$ & 1.71 & \pm 0.29 (stat + syst $)$ \\
$\sigma(\nu)$ & 7.07 & \pm 1.20 (stat + syst $)$ \\
$R(\nu, \bar{\nu})$ & Ratios & \\
\hline \hline
\end{tabular}

its value depends on the particular experiment and data taking periods. The event rate ratio $r(\nu, \bar{\nu})$ fractional systematic uncertainty is the same as cross section ratio $R(\nu, \bar{\nu})$, except it does not include the flux errors given in Table III. The fractional systematic errors are $1.92 \%$ for the restricted phase space selections.

\section{B. Discussion of results}

In this section, we discuss how our results compare with NEUT predictions, previous measurements, the impact on future CPV measurements and the multinucleon effects that can modify neutrino cross sections.

We observe close agreement between the numbers of data events and the NEUT MC generated events in both the unrestricted and restricted phase space selected events. Using Table VI, the data to $\mathrm{MC}$ ratios for the restricted phase space selection for the FHC/RHC modes are $94.2 \% / 91.7 \%$.

We can compare our neutrino result to previous T2K publications that used the FGD subdetector with a scintillator target. The previous T2K flux averaged CCINC [6] was $\quad(6.91 \pm 0.13$ (stat) \pm 0.84 (syst) $) \times 10^{-39} \mathrm{~cm}^{2}$ per nucleon and this is within systematic errors to our full phase space measurement in Table VIII. The published T2K CCQE [7] and events of the charged current process that has no pions $(\mathrm{CC} 0 \pi)$ [36] flux averaged cross sections per nucleon are $(3.83 \pm 0.55) \times 10^{-39} \mathrm{~cm}^{2}$ and $(4.17 \pm 0.05 \pm 0.47) \times 10^{-39} \mathrm{~cm}^{2}$, respectively. In the context of the NEUT model, the CCINC results presented here are compatible with the CCQE and CCOpi results from these prior publications. These full phase space neutrino results agree with the previous $\mathrm{T} 2 \mathrm{~K}$ measurements.

The near detector flux averaged uncertainties on the ratio of cross sections and rates are useful to estimate the sensitivity of future $C P$ conservation tests in long baseline appearance experiments. The restricted phase space fractional systematic errors on $R(\nu, \bar{\nu})$ and $r(\nu, \bar{\nu})$ are $4.0 \%$ and $1.8 \%$, respectively. These systematic errors on the near detector ratio measurements are now due to many small errors less than $1 \%$, so further substantial improvements will be challenging. Although future measurements of appearance probabilities are likely to be limited by statistical uncertainties on far detector $\nu_{e}$ and $\bar{\nu}_{e}$ measurements, the near detector uncertainties on $\nu_{\mu}$ and $\bar{\nu}_{\mu}$ measurements may also limit the ultimate precision of future CPV tests.

The $2 \mathrm{p} 2 \mathrm{~h}$ models have been predicted [11] to affect the difference between the $\nu_{\mu}$ and $\bar{\nu}_{\mu}$ cross sections. The NEUT MC predictions of the $\nu_{\mu}$ and $\bar{\nu}_{\mu}$ cross sections, their difference and sum, their ratio, and their asymmetry have been calculated in four models: (1) NEUT with a default spectral function [37], (2) RFG model, (3) RFG model with RPA corrections and (4) RFG with RPA corrections and $2 \mathrm{p} 2 \mathrm{~h}$ interactions. The MC model (4) included $2 \mathrm{p} 2 \mathrm{~h}$ effects in the NEUT MC generator from the model by Nieves [12], and this model (4) was also used to calculate the Table VI 
TABLE IX. The numerical values of model 3 predictions and the corresponding measurements shown in Figs. 7 and 8.

\begin{tabular}{lcccccc}
\hline \hline Model 3 & $\sigma(\bar{\nu})$ & $\sigma(\nu)$ & $\Delta(\nu, \bar{\nu})$ & $\Sigma(\nu, \bar{\nu})$ & $R(\nu, \bar{\nu})$ & $A(\nu, \bar{\nu})$ \\
\hline MC predictions & 0.908 & 2.36 & 1.45 & 3.26 & 0.385 & 0.444 \\
Measurements & $0.911 \pm 0.094$ & $2.45 \pm 0.24$ & $1.55 \pm 0.16$ & $3.37 \pm 0.33$ & $0.371 \pm 0.019$ & $0.459 \pm 0.021$ \\
\hline
\end{tabular}

and VII results. The six cross section and ratio measurements (solid circles with error bars) are presented in six plots in Figs. 7 and 8. In each plot, the four different model predictions (open squares) are compared for the same measurement. Note each model can have slightly different efficiencies, so the corresponding measurement corrected for efficiency can vary depending upon the particular model. These models include additional nuclear effects such as $2 \mathrm{p} 2 \mathrm{~h}$ that make different predictions for neutrino and antineutrino enhancements to the cross section. We find different cross section combinations can help differentiate the models and here we investigate a limited number of model combinations available in NEUT. The measured cross sections are stable and have negligible changes with different models. This demonstrates the efficiencies are similar in different models. The observed $\bar{\nu}_{\mu}$ cross section has slightly better agreement with model 3 ; however, the other models 1, 2 and 4 predictions are nearly all within 1 standard deviation of the data uncertainties. The numerical values of the model 3 predictions and the data results are given in Table IX. Although the uncertainty on our model combinations is relatively large, it is clear that with higher statistics, such comparisons will be valuable for model separation.

In future $\mathrm{T} 2 \mathrm{~K}$ measurements, more statistics, especially in the $\bar{\nu}_{\mu}$ mode, will enable differential water subtracted measurements in bins of muon momentum and angle. After unfolding, the differential measurements of ratios in
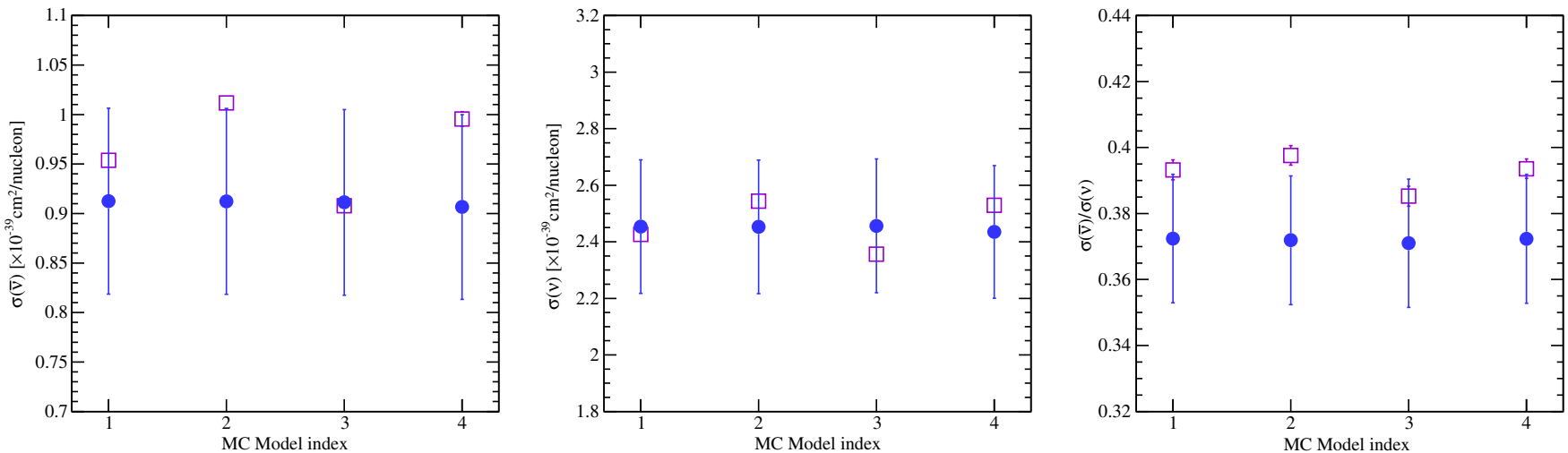

FIG. 7. Comparison of MC model 1-4 predictions, open squares with no errors bars, to data results, solid circles with error bars, in measurements of cross sections $\sigma\left(\bar{\nu}_{\mu}\right)$ [left] and $\sigma\left(\nu_{\mu}\right)$ [middle] and the $R$ ratio $\sigma\left(\bar{\nu}_{\mu}\right) / \sigma\left(\nu_{\mu}\right)$ [right].
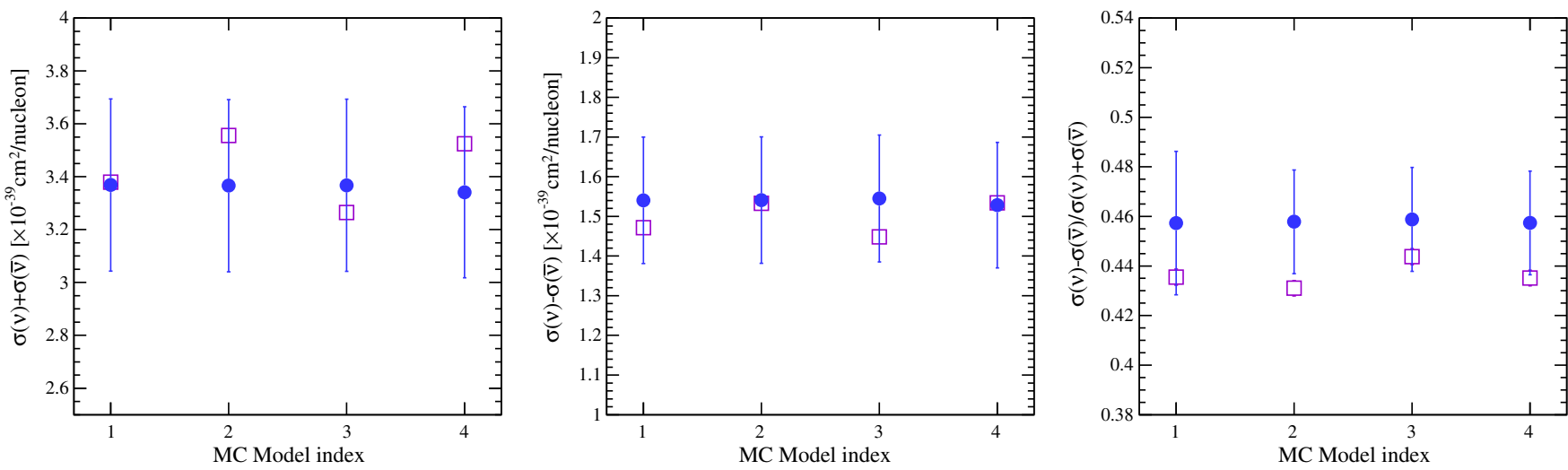

FIG. 8. Comparison of MC model 1-4 predictions, open squares with no errors bars, to data results, solid circles with error bars, in measurements of the cross section sum $\Sigma=\sigma\left(\nu_{\mu}\right)+\sigma\left(\bar{\nu}_{\mu}\right)$ [left], difference $\Delta=\sigma\left(\nu_{\mu}\right)-\sigma\left(\bar{\nu}_{\mu}\right)$ [middle] and asymmetry $A=$ $\left(\sigma\left(\nu_{\mu}\right)-\sigma\left(\bar{\nu}_{\mu}\right)\right) /\left(\sigma\left(\nu_{\mu}\right)+\sigma\left(\bar{\nu}_{\mu}\right)\right)[$ right $]$. 
particular, differences and sums are expected to provide improved estimates of systematic uncertainties in future experimental CPV tests and better tests of $2 \mathrm{p} 2 \mathrm{~h}$ models.

\section{CONCLUSIONS}

In summary, the $\mathrm{T} 2 \mathrm{~K}$ experiment has measured charged current inclusive events, in a restricted phase space of $\theta_{\mu}<32^{\circ}$ and $p_{\mu}>500 \mathrm{MeV} / \mathrm{c}$, the flux averaged cross sections $\left(\mathrm{cm}^{2}\right.$ per nucleon) and ratio of cross sections, as

$$
\begin{aligned}
& \sigma(\bar{\nu})=(0.900 \pm 0.029(\text { stat }) \pm 0.088(\text { syst })) \times 10^{-39}, \\
& \sigma(\nu)=(2.41 \pm 0.021(\text { stat }) \pm 0.231(\text { syst })) \times 10^{-39}
\end{aligned}
$$

and

$$
R\left(\frac{\sigma(\bar{\nu})}{\sigma(\nu)}\right)=0.373 \pm 0.012(\text { stat }) \pm 0.015(\text { syst }) .
$$

The $\bar{\nu}_{\mu}$ inclusive cross section and the ratio $R$ results are the first published measurements at $\nu_{\mu}$ and $\bar{\nu}_{\mu}$ flux energies [38] below $1.5 \mathrm{GeV}$. Although the current uncertainty on the different model combinations is relatively large, we expect future higher statistics comparisons will be valuable for model discrimination.

\section{ACKNOWLEDGMENTS}

We thank the J-PARC staff for superb accelerator performance. We thank the CERN NA61/SHINE Collaboration for providing valuable particle production data. We acknowledge the support of MEXT, Japan; NSERC (Grant No. SAPPJ-2014-00031), NRC and CFI, Canada; CEA and CNRS/IN2P3, France; DFG, Germany; INFN, Italy; National Science Centre (NCN) and Ministry of Science and Higher Education, Poland; RSF, RFBR, and MES, Russia; MINECO and ERDF funds, Spain; SNSF and SERI, Switzerland; STFC, UK; and DOE, USA. We also thank CERN for the UA1/NOMAD magnet, DESY for the HERA-B magnet mover system, NII for SINET4, the WestGrid and SciNet consortia in Compute Canada, and GridPP in the United Kingdom. In addition, participation of individual researchers and institutions has been further supported by funds from ERC (FP7), H2020 Grant No. RISE-GA644294-JENNIFER, EU; JSPS, Japan; Royal Society, UK; the Alfred P. Sloan Foundation and the DOE Early Career program, USA.
[1] Y. Fukuda et al. (Super-Kamiokande Collaboration), Phys. Rev. Lett. 81, 1562 (1998).

[2] For a description of neutrino physics see reviews in $\mathrm{C}$. Patrignani et al. (Particle Data Group), Chin. Phys. C 40, 100001 (2016). The neutrino parameters are given in section 14. The $\mathrm{CP}$ violations tests are given in section 14.7 . The matter effects are given in section 14.8.

[3] S. P. Mikheev and A. Yu. Smirnov, Sov. J. Nucl. Phys. 42, 913 (1985); L. Wolfenstein, Phys. Rev. D 17, 2369 (1978).

[4] K. Abe et al. (T2K Collaboration), Nucl. Instrum. Methods Phys. Res., Sect. A 659, 106 (2011); P. Adamson et al. (NOvA Collaboration), Nucl. Instrum. Methods Phys. Res., Sect. A 806, 279 (2016); K. Anderson et al., The NuMI Facility Technical Design Report, FNAL Laboratory Report Fermilab-Design-1998-01 (unpublished).

[5] R. Acciarri et al. (DUNE Collaboration), arXiv:1601.02984; K. Abe et al. (HyperK Collaboration), KEK preprint 2016-21, https://lib-extopc.kek.jp/preprints/PDF/2016/1627/ 1627021.pdf (unpublished).

[6] K. Abe et al., Phys. Rev. D 87, 092003 (2013). Note that this publication used the FGD detector which is mainly a hydrocarbon target, whereas this paper reports on a target that is mainly water + hydrocarbon with heavier elements given in Table 1.

[7] K. Abe et al., Phys. Rev. D 92, 112003 (2015). Note that this publication quotes the cross section per neutron. In this paper we provide the CCQE cross section as $(4.15 \pm 0.6) \times$ $10^{-39} \mathrm{~cm}^{2}$ per nucleon which is a factor 2 smaller.
[8] L. Ren et al. (MINVERVA Collaboration), Phys. Rev. D 95, 072009 (2017).

[9] A. Aguilar-Arevalo et al. (MiniBooNE Collaboration), Phys. Rev. D 81, 092005 (2010); A. Aguilar-Arevalo et al., Phys. Rev. D 88, 032001 (2013).

[10] M. Martini, M. Ericson, G. Chanfray, and J. Marteau, Phys. Rev. C 81, 045502 (2010).

[11] M. Ericson and M. Martini, Phys. Rev. C 91, 035501 (2015).

[12] R. Gran, J. Nieves, F. Sanchez, and M. J. Vicente Vacas, Phys. Rev. D 88, 113007 (2013).

[13] C. Juszczak, J. T. Sobczyk, and J. Zmuda, Phys. Rev. C 82, 045502 (2010); J. Nieves, I. R. Simo, and M. V. Vacas, Phys. Lett. B 707, 72 (2012).

[14] T2K Experiment, K. Abe et al. (T2K Collaboration), Nucl. Instrum. Methods Phys. Res., Sect. A 659, 106 (2011).

[15] K. Abe et al. (T2K Collaboration), Phys. Rev. D 87, 012001 (2013); 87, 019902 (2013).

[16] FLUKA package, G. Battistoni, S. Muraro, P. R. Sala, F. Cerutti, A. Ferrari, S. Roesler, A. Fasso, and J. Ranft, AIP Conf. Proc. 896, 31 (2007); A. Ferrari, P. R. Sala, A. Fasso, and J. Ranft, CERN Report CERN- 2005-010, SLAC Report SLAC-R-773 and INFN Report INFN-TC-05-11 (unpublished reports).

[17] S. Agostinelli et al. (GEANT4 collaboration), Nucl. Instrum. Methods Phys. Res., Sect. A 506, 250 (2003).

[18] GCALOR package, C. Zeitnitz, and T. A. Gabriel, Nucl. Instrum. Methods Phys. Res., Sect. A 349, 106 (1994). 
[19] N. Abgrall et al. (NA61/SHINE Collaboration), Eur. Phys. J. C 76, 84 (2016).

[20] See the detailed descriptions and the references for TPC analysis selections in Sec. IV., for neutrino interactions in Sec. V., for beam flux uncertainties in Sec. VI A. and for CC-other in Sec. VI. B. in K. Abe et al. (T2K Collaboration), Phys. Rev. D 92, 112003 (2015).

[21] S. Assylbekov et al. (T2K ND280 PøD Collaboration), Nucl. Instrum. Methods Phys. Res., Sect. A 686, 48 (2012).

[22] N. Abgrall et al. (T2K ND280 TPC Collaboration), Nucl. Instrum. Methods Phys. Res., Sect. A 637, 25 (2011).

[23] K. Abe et al. (T2K Collaboration), Phys. Rev. D 91, 112010 (2015).

[24] The NEUT simulation is described in Y. Hayato, Acta Phys. Pol. B 40, 2477 (2009). This version 5.3.2 includes the multinucleon ejection model and nuclear long-range correlations for CCQE interactions treated in the random phase approximation.

[25] J. A. Formaggio and G. P. Zeller, Rev. Mod. Phys. 84, 1307 (2012).

[26] Previous T2K published analyses which include extensive descriptions and use of the interaction model include K. Abe et al. (T2K Collaboration), Phys. Rev. D 93, 112012 (2016); K. Abe et al. (T2K Collaboration), Phys. Rev. D 92, 112003 (2015).

[27] C. H. Llewellyn Smith, Phys. Rep. 3, 261 (1972).

[28] R. Bradford, A. Bodek, H. S. Budd, and J. Arrington, Nucl. Phys. B, Proc. Suppl. 159, 127 (2006).

[29] R. A. Smith and E. J. Moniz, Nucl. Phys. B43, 605 (1972); B101, 547(E) (1975).
[30] J. Nieves, I. R. Simo, and M. J. Vicente Vacas, Phys. Rev. C 83, 045501 (2011).

[31] D. Rein and L. M. Sehgal, Ann. Phys. (N.Y.) 133, 79 (1981).

[32] K. M. Graczyk and J. T. Sobczyk, Phys. Rev. D 77, 053001 (2008); K. M. Graczyk and J. T. Sobczyk, Phys. Rev. D 79, 079903(E) (2009).

[33] M. Gluck, E. Reya, and A. Vogt, Eur. Phys. J. C 5, 461 (1998).

[34] A. Bodek and U. K. Yang, AIP Conf. Proc. 670, 110 (2003).

[35] The recent published $M_{A}^{\mathrm{QE}}$ measurements from the $\mathrm{K} 2 \mathrm{~K}$, MiniBooNE, MINOS, and T2K experiments in $\mathrm{GeV} / \mathrm{c}^{2}$ are $1.20 \pm 0.12,1.35 \pm .17,1.23_{-.09}^{+.13}(\text { stat })_{-.15}^{+.12}$ (syst) and $1.26_{-.18}^{+.21}$, respectively. These results have been published in R. Gran et al. (K2K Collaboration), Phys. Rev. D 74, 052002 (2006); A. Aguilar-Arevalo et al. (MiniBooNE Collaboration), Phys. Rev. D 81, 092005 (2010); P. Adamson et al. (MINOS Collaboration), Phys. Rev. D 91, 012005 (2015); K. Abe et al. (T2K Collaboration), Phys. Rev. D 92, 112003 (2015); The bubble chamber results $\left(M_{A}=0.94 \pm .03 \mathrm{GeV} / \mathrm{c}^{2}\right)$ have been reanalyzed in $\mathrm{K}$. M. Graczyk, D. Kielczewska, P. Przewlocki, and J. T. Sobczyk, Phys. Rev. D 80, 093001 (2009).

[36] K. Abe et al. (T2K Collaboration), Phys. Rev. D 93, 112012 (2016).

[37] O. Benhar and A. Fabrocini, Phys. Rev. C 62, 034304 (2000).

[38] See http://t2k-experiment.org/results/neutrino-beam-fluxprediction-2016/ for the detailed ND280 FHC and RHC neutrino/antineutrino flux predictions used in this paper. 